\title{
Wave activity (planetary, tidal) throughout the middle atmosphere (20-100 km) over the CUJO network: Satellite (TOMS) and Medium Frequency (MF) radar observations
}

\author{
A. H. Manson ${ }^{1}$, C. E. Meek ${ }^{1}$, T. Chshyolkova ${ }^{1}$, S. K. Avery ${ }^{2}$, D. Thorsen ${ }^{3}$, J. W. MacDougall ${ }^{4}$, W. Hocking ${ }^{4}$, \\ Y. Murayama ${ }^{5}$, and K. Igarashi ${ }^{5}$ \\ ${ }^{1}$ Institute of Space and Atmospheric Studies, University of Saskatchewan, 116 Science Place, Saskatoon, SK, S7N 5E2, \\ Canada \\ ${ }^{2}$ CIRES, University of Colorado, Boulder, USA \\ ${ }^{3}$ Department of Electrical and Computer Engineering, University of Alaska, Fairbanks, USA \\ ${ }^{4}$ Department of Physics and Astronomy, University of Western Ontario, London, Canada \\ ${ }^{5}$ National Institute of Information and Communications Technology, Tokyo, Japan
}

Received: 12 February 2004 - Revised: 12 November 2004 - Accepted: 6 December 2004 - Published: 28 February 2005

\begin{abstract}
Planetary and tidal wave activity in the tropopause-lower stratosphere and mesosphere-lower thermosphere (MLT) is studied using combinations of groundbased (GB) and satellite instruments (2000-2002). The relatively new MFR (medium frequency radar) at Platteville $\left(40^{\circ} \mathrm{N}, 105^{\circ} \mathrm{W}\right)$ has provided the opportunity to create an operational network of middle-latitude MFRs, stretching from $81^{\circ} \mathrm{W}-142^{\circ} \mathrm{E}$, which provides winds and tides 70 $100 \mathrm{~km}$. CUJO (Canada U.S. Japan Opportunity) comprises systems at London $\left(43^{\circ} \mathrm{N}, 81^{\circ} \mathrm{W}\right)$, Platteville $\left(40^{\circ} \mathrm{N}\right.$, $\left.105^{\circ} \mathrm{W}\right)$, Saskatoon $\left(52^{\circ} \mathrm{N}, 107^{\circ} \mathrm{W}\right)$, Wakkanai $\left(45^{\circ} \mathrm{N}\right.$, $\left.142^{\circ} \mathrm{E}\right)$ and Yamagawa $\left(31^{\circ} \mathrm{N}, 131^{\circ} \mathrm{E}\right)$. It offers a significant $7000-\mathrm{km}$ longitudinal sector in the North American-Pacific region, and a useful range of latitudes $\left(12-14^{\circ}\right)$ at two longitudes. Satellite data mainly involve the daily values of the total ozone column measured by the Earth Probe (EP) TOMS (Total Ozone Mapping Spectrometer) and provide a measure of tropopause-lower stratospheric planetary wave activity, as well as ozone variability.

Climatologies of ozone and winds/tides involving frequency versus time (wavelet) contour plots for periods from 2-d to 30-d and the interval from mid 2000 to 2002, show that the changes with altitude, longitude and latitude are very significant and distinctive. Geometric-mean wavelets for the region of the $40^{\circ} \mathrm{N}$ MFRs demonstrate occasions during the autumn, winter and spring months when there are similarities in the spectral features of the lower atmosphere and at mesopause $(85 \mathrm{~km})$ heights. Both direct planetary wave (PW) propagation into the MLT, nonlinear PW-tide interactions, and disturbances in MLT tides associated with fluctuations in the ozone forcing are considered to be possible coupling processes. The complex horizontal wave numbers
\end{abstract}

Correspondence to: A. H. Manson

(alan.manson@usask.ca) of the longer period oscillations are provided in frequency contour plots for the TOMS satellite data to demonstrate the differences between lower atmospheric and MLT wave motions and their directions of propagation.

Key words. Meteorology and atmospheric dynamics (Middle atmosphere dynamics; Synoptic-scale meteorology; Waves and tides)

\section{Introduction}

Studies of the dynamics of the atmosphere involving the lower and middle atmosphere are comparatively rare, at least partly due to the limited range of sensitivity of many sounding instruments, but also due to the interests of the authors. Some of our early papers considered the winds MLT (60$100 \mathrm{~km})$ and the association of their variability with stratospheric processes, e.g. Gregory and Manson (1975); and much more recently, Hoffmann et al. (2002) and Manson et al. (2002a) assessed the coupling between stratospheric winter warming events (using radiosonde and satellite sensors) and MLT winds, gravity waves (GW) and tides using MFR radars. Variability of stratospheric temperatures with scales of several days has also been associated with variability of MLT tides and winds (Manson et al., 1982), but remarkably little has been done since then.

A more recent study worthy of note is that by Lawrence and Randel (1996), who used Nimbus 6 satellite data (PMR) to demonstrate stratospheric-mesospheric coupling in three distinctive ways: the variance of the geopotential height around latitude circles $\left(50-70^{\circ} \mathrm{N}\right)$ showed strong correlation throughout the atmosphere $(35-83 \mathrm{~km})$; and episodic wave-like events, in particular westward propagating "normal" PW modes (periods, 5 to 10-d band) having near global 
coherence, and eastward propagating PWs (4-d period) in southern winters (hereafter $\mathrm{SH}$, Southern Hemisphere) associated with an instability process, were identified throughout the atmosphere.

Most recently, and during the preparation of this paper, there have been three papers published which have interested the authors and inevitably led to some of the directions pursued in our work here. Pogoreltsev et al. (2002) identified a 6.5-d PW of wave number one $(\mathrm{m}=1)$ in the MLT using the Saskatoon $\left(52^{\circ} \mathrm{N}\right)$ and Sheffield $\left(53^{\circ} \mathrm{N}\right)$ MFR and Meteor radars, and explained this wave in terms of nonlinear interactions between the normal mode $(\mathrm{m}=2)$ of period 7- $\mathrm{d}$ and the standing PW (SPW) with $\mathrm{m}=1$. UKMO (United Kingdom Meteorological Office) assimilated stratospheric fields and a 2-D numerical model were used for explanatory purposes within the study. A more general study by Fedulina et al. (2002) of the stratospheric PW at 1hPa using UKMO "data" preceded this work: longer period PW $(\mathrm{T}>10$-d) were dominant during winter months in both hemispheres ( $\mathrm{SH}$, $\mathrm{NH}$ ), with eastward and westward propagating waves being seen; in the $\mathrm{SH}$ at wavenumber one $(\mathrm{m}=1)$ eastward waves had somewhat larger amplitudes, while westward wave activity was seasonally more sustained or of larger amplitude in the NH. Shorter period waves (4- to 5-d) were dominantly westward propagating and while they were seen throughout the year, they increased in amplitude during the seasonal transitions.

Pancheva et al. (2003) assessed the variability of the semidiurnal (12-h) tide (circa $92 \mathrm{~km}$ altitude) due to fluctuations in solar activity and total (column) ozone. The latter is of interest to us here. They provided frequency spectra of TOMS data (Total Ozone Mapping Spectrometer) and Meteor radar semidiurnal tidal amplitude data (Sheffield, $53^{\circ} \mathrm{N}$ ) for the entire autumn to spring intervals of 4 years (19891993), which then showed similarities in the bands of 8 $12,15-18$ and 25-28 day oscillations; the cross products of the respective wavelet spectra showed strong features, especially near 16 and 27-28 days, which were linked in their occurrences (1989-1993) to the QBO (Quasi Biennial Oscillation), stratospheric warmings, and solar variability. Finally, Lawrence and Jarvis (2003) assessed the austral winter (1997-1999) PW activity in the stratosphere (the European Centre for Medium range Weather Forecasting (ECMWF) wind product at $30 \mathrm{~km}$ ), upper middle atmosphere (an HF radar (interferometer) provided winds $75-95 \mathrm{~km}$ ) and thermosphere (the HF radar operated as an ionosonde). The radar is at Halley Bay, 76S. Their results were complex and to some degree apparently inconsistent with the Northern Hemisphere study: there were times when strong PW activity occurred in either the stratosphere or middle-upper atmosphere, with no simultaneous activity in the other region; perversely, evidence for PW activity in the mesosphere was stronger when the stratospheric activity was more restricted in latitudinal extent; and a quite consistent anti-correlation existed between PW activity in the mesosphere and the Eregion. Processes suggested included re-generation of the PW component at higher altitudes through gravity wave
(GW) filtering and breaking, and nonlinear interactions between PW and tides.

In this study we use the Earth probe (EP) TOMS data (level-3 product) for the time interval mid-2000 to 2002, and wind data from the 5 MFR radars of the CUJO network (London, Platteville, Saskatoon, Wakkanai and Yamagawa). Wavelet analysis (a swept frequency spectral analysis), as described briefly below, is applied to TOMS and the MFR wind data to assess PW activity and propagation throughout the middle atmosphere. It is then also applied to TOMS and the MFR tidal amplitude data (diurnal and semidiurnal) to assess the variability of tidal forcing and/or propagation processes at periods consistent with PW variability. It is appropriate at this point to mention the very useful and insightful paper by Schoeberl and Krueger (1983) that was brought to our attention by Randel (private communication, 2003). They demonstrated, using SH data, that the variability of the total ozone (column) parameter is a very useful diagnostic for wave-like (PW) disturbances of medium zonal wave numbers. This is based upon the slowness of photochemical processes in the lower stratosphere and below, such that the ozone may be considered a passive tracer. Their conclusion was that, because the meridional and vertical advection of ozone correlates strongly, evanescent waves near the tropopause produce the maximum ozone (column) signal. They studied examples of these waves, including an $\mathrm{m}=5$ eastward propagating $\mathrm{PW}$ and also synoptic scale baroclinic waves (also eastward moving) which decay above the tropopause (Charney and Drazin, 1961). They noted that the winter ozone fluctuations of the $\mathrm{NH}$ are a complex superposition of disturbances from mesoto planetary scale, while the SH disturbances are more regular (hence their detailed study of the $\mathrm{m}=5 \mathrm{PW}$ ); and also that propagating winter waves $(\mathrm{m}=1,2)$ will provide a more complex signal in the total ozone, as the advection terms will not act to strengthen the signal in the total ozone. It will be shown here that wave number spectral analysis of the TOMS data distinguish these eastward propagating PW very clearly. We shall also use cross products of the wavelet spectra (for individual locations and also regions) to emphasize the similarities of variability at PW periods, and the coupling, between the stratosphere and mesosphere. There is also discussion of the present and future use of UKMO dataassimilation products, which includes radiosonde and satellite data, and allows for analysis to near $50 \mathrm{~km}$ in the middle atmosphere.

\section{Systems and data analysis}

\subsection{Radars}

The Saskatoon, London and Platteville MFRs (2.2 MHz) are of very similar design, and have been well described in the first CUJO papers (Manson et al., 2003a, 2004a). The spaced antenna "full correlation analysis" method is used (Meek, 1980). Vertical soundings provide wind sampling at $3 \mathrm{~km}$ intervals every 5 min from near 60 to $100 \mathrm{~km}$. The MFRs are of 


\section{Saskatoon Zonal Daily Mean Wind (solid) vs. TOMS DU (dashed) for 2001}

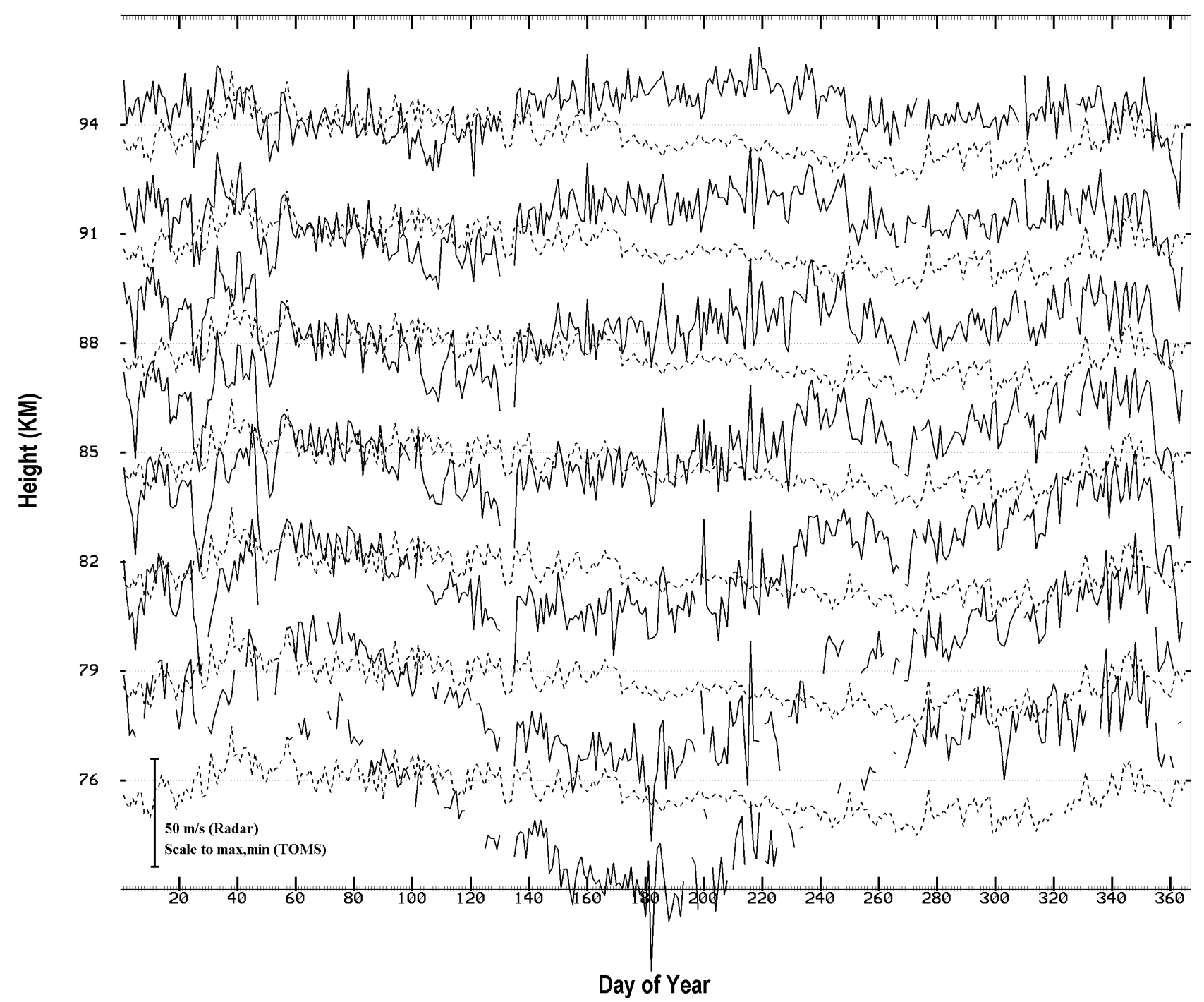

Fig. 1. Time sequences of daily mean zonal (east-west, EW) winds from the MF radar at Saskatoon $\left(52^{\circ} \mathrm{N}\right)$ for heights from $76-94 \mathrm{~km}$ : the distance between the $3 \mathrm{~km}$ samples represents $50 \mathrm{~m} / \mathrm{s}$. The dashed lines are for TOMS values (median DU value in a $5^{*} 5$ grid point cell centred on Saskatoon): these are arbitrarily scaled according to the maximum variation during the year. The TOMS sequences are repeated at each MFR height.

basically similar configuration, and any differences will not lead to differences in the measured winds; e.g. the MFRs in Japan are Australian ATRAD systems (Igarashi et al., 1996; Murayama et al., 2000), which although physically very similar to the Canadian systems, use a more classical method of analysis which is closer to that described by Briggs (1984). Comparisons have shown that no significant differences exist between these methods (Thayaparan et al., 1995a). The Japanese radars provide samples of wind every 2 rather than $3 \mathrm{~km}$, and 2 rather than $5 \mathrm{~min}$, on a continuous basis. Our earlier detailed studies of PW (Luo et al., 2000, 2002a, 2002b) demonstrated that amplitudes of $>3 \mathrm{~m} / \mathrm{s}$ from spectral analyses provided a geophysically realistic signal.
All wind measurement systems have biases or selectivity issues, so comments upon these for the radar winds are useful. Examples of significant published comparisons include an MFR and Meteor Wind Radar (MWR) at $43^{\circ} \mathrm{N}$ (Thayaparan and Hocking, 2002); MFRs and Fabry-Perot Interferometers ("green line" and hydroxyl) at two $52^{\circ} \mathrm{N}$ locations (Manson et al., 1996; Meek et al., 1997); and rockets and radars (MFR, VHF and EISCAT) near $70^{\circ} \mathrm{N}$ (Manson et al., 1992). Most recently, there has also been an extensive comparison (Manson et al., 2004b) for the year 2000 involving two MFRs and an MWR, which comprise the so-called Scandinavian-Triangle $\left(68-70^{\circ} \mathrm{N}\right.$, radar spacings of $125-$ $270 \mathrm{~km}$ ). In these studies the directions of the winds or their 
perturbations (tidal phases) have been satisfactorily consistent e.g. means within standard errors. However, while the Saskatoon optical-radar comparisons showed no speed biases, a limited "SKiYMET" MWR-MFR campaign at Saskatoon (spring-summer, 1998) showed modest differences, e.g. MFR/MWR ratios of about 0.75 near 88 km (Meek, Hocking and Manson, private communication, 2002); at $70^{\circ} \mathrm{N}$, Troms $\varnothing$, the speeds from the MFR were 0.65 of those from the other types of radar and rocket systems for heights from $82-97 \mathrm{~km}$; and the Scandinavian-Triangle provided median MFR/MWR ratios of $0.91 / 0.63$ at $85 / 97 \mathrm{~km}$. Finally, systematic differences between "satellite-winds" from HRDIUARS (Doppler Imager) and radars have also been noted, e.g. Saskatoon MFR speeds are $0.75-0.8$ of HRDI speeds (Meek et al., 1997). For our purposes here, and especially as we are using only MFR radars for the upper middle atmosphere, we will simply bear in mind the potential speed biases when any later comments are made on wave amplitudes.

\subsection{Analysis techniques}

To provide information on atmospheric oscillations from 2$\mathrm{d}$ to 30-d at particular heights (Sect. 3), a wavelet analysis has been applied to the years of daily mean winds, tides (harmonic analyses described below) or TOMS data, with additional data at the ends being used to cover the full sliding window for all wave periods used (Figs. 2-6). A Gaussian window of length 6 times the period (truncated at 0.05 of peak value) is used to approximate a Morlet wavelet analysis (Kumar and Foufoula-Georgiou, 1997), but one in which gaps do not have to be filled. A Fourier transform (not an FFT) is therefore used and applied to existing data points only. Data selection criteria require that $80 \%$ of the phase values in two different periods are available. Breaks in the heavy data-existence lines at the bottoms of the frames indicate that there were no data for those days, and provide a warning that spectral data near the edges of and during these intervals may be inaccurate. The period-scale uses 600 pixels and is linear in $\log$ (period) from 2 to 31 days. Each pixel represents one spectral value, and there is no smoothing.

Amplitudes are increased appropriately during the calculations to correct for attenuation by the window, but on the assumption that there are no significant gaps. In the plots the value in $\mathrm{dB}$ (decibels) is equal to $20 \log _{10}$ (wave amplitude in $\mathrm{m} / \mathrm{s}$ for the MFR). This scale is used because of the large range of amplitudes that exist for the various types of waves (tides to PW) in the MLT region. Color renditions are optimized by this scaling. There is discussion of significance levels for the more complex figures later, near their presentation, but for the basic wavelet figures significances have been taken to be as in Luo et al. (2002a). There they were determined by comparison with "Lomb-Scargle" periodograms (Lomb, 1976; Scargle, 1982; Luo et al., 2002a), for which significance levels are available. Based upon these, $\mathrm{dB}$ circa $17.5(7 \mathrm{~m} / \mathrm{s}$, yellow) can be considered significant at the $99 \%$ level.
Tidal amplitudes are produced by analyzing 2-day sequences shifted by $12 \mathrm{~h}$. Data must be available for $12 \mathrm{~h}$ on each of the 2 days, following which a Fourier transform is done. Amplitudes at periods of 48, 24, 12, $8 \mathrm{~h}$ are used to order these components, which are then fitted separately by least-squares fit and subtracted in order of size, weith the largest first. For the wavelet analysis, time sequences of amplitudes are used (with samples every $12 \mathrm{~h}$ ), but the periods displayed start at 2-d (rather than the Nyquist period of 1-d) to match the other analyses.

The daily background wind parameter comes from a leastsquares fit of mean, 24-h and 12-h oscillations to single days. There must be data in at least $16 \mathrm{~h}$ of each day for the fitting to proceed. These background or mean winds are used in Fig. 1 and in the wavelet analyses. (The combination of different analyses and wavelet selection criteria lead to apparent, but unimportant, differences in data gap locations between background winds and tidal amplitudes in the figures.)

Geometric means have been used as a type of "crosswavelet" analysis (Figs. 5 and 6). These are the means of wavelet amplitudes and do not involve the wavelet phases. The geometric mean wavelets are useful in that all parameters must be relatively large for a large output, but there is no certainty that a high value refers to a coherent oscillation. However, there is discussion of the phases of the wavelets in later sections. The motivation for forming these "regional" means (for the CUJO network of North American-Japanese $40^{\circ} \mathrm{N}$ locations) is due to the intermittency, both spatial and temporal, found in our earlier studies of PW (Luo et al., 2002a; Manson et al., 2004a).

Wave number analysis of TOMS data (Figs. 7 and 8) is as follows: a 60-day time sequence is selected and then detrended. This latter is done by fitting a mean plus an annual sine wave to 365 days of longitudinally averaged values, and then subtracting these from each longitude value (the "bins" are spaced by $\left.1.25^{\circ}\right)$. Then frequency spectra (80\% existence criterion is required) are calculated for each 60-day detrended sequence and coherently averaged over a band of 5 TOMS latitudes (this is then a $5^{\circ}$ average). In this spectral analysis the sampling times are pre-adjusted to refer to the same absolute time at different longitudes. This then provides, for any chosen 60-day interval, complex amplitudes at each frequency for each longitude bin. The sequence of complex amplitudes versus longitude is then Fourier transformed, resulting in amplitudes at positive and negative spatial wave numbers. We use the convention that a positive wave number indicates westward propagation, as is frequently done for tidal analysis, e.g. Manson et al., 2004c. A log scale is convenient for showing a large range of amplitudes, and even though $\mathrm{dB}\left(20 \log _{10} \mathrm{rms} \mathrm{DU}\right.$ (Dobson Units)) is a power unit and not normally applicable to total ozone, it is a well understood unit. 


\section{Comparisons of Tropopause-Stratospheric (TOMS) and Mesospheric/MLT Data}

\subsection{Variability of Total Ozone and Mesospheric Winds at CUJO sites}

Given the potential complexity of the linkages between the different atmospheric regions, and indeed the different parameters being used, we will mention and show a variety of comparisons which range from simple time sequences and their correlations, to harmonic means (cross-products) of individual wavelets. Time sequences of the daily mean zonal winds (eastward being positive) during the year 2001 are plotted in Fig. 1 for the Saskatoon MFR; heights from 76$94 \mathrm{~km}$ are provided, along with the TOMS data (the units being Dobson Units, $300 \mathrm{DU}$ is equivalent to a $3-\mathrm{mm}$ column of ozone) for that location. The latter are medians in a $5 * 5$ degree cell area centred on Saskatoon. This figure provides substantial information. At the lowest heights the summer winds are near $-50 \mathrm{~m} / \mathrm{s}$, which are consistent with the stratospheric-mesospheric mid-latitude westward jet, while in winter the circa $40 \mathrm{~m} / \mathrm{s}$ values are consistent with the winter eastward jet. Frequently, planetary waves of the troposphere and stratosphere will not propagate through this strong summer westward jet due to critical levels (wave phase speed matching the background wind speed) being achieved; and indeed, a strong winter eastward jet will also inhibit propagation of the larger $(m>3$ or 4$)$ wave number modes (Charney and Drazin, 1961; Luo et al., 2002a; Manson et al., 2003a, 2004a) due to their having small "critical speeds" (this parameter varies as the inverse square of the horizontal wave numbers). Thus, strong differences between the oscillatory periods (and their amplitudes) of the winds, associated with PW, in the MLT region are expected for the two solstices.

Such is indeed the case, based upon visual inspection of Fig. 1 and the wavelet spectra which follow. Wind oscillations of near $20 \mathrm{~m} / \mathrm{s}$ amplitude and within a 10 - to 30 -d period are clearly evident from $76-94 \mathrm{~km}$ during winter and autumn months (exhibiting little phase shift with height), while the amplitudes are smaller and the periods shorter (e.g. 2-d oscillations are evident) during the spring and summer. The TOMS data share a rather similar morphology, although the summer variability is comparatively less. There are also very modest visible indications of correlations between the TOMS and MFR winds data during the autumn and winter e.g. the $91 \mathrm{~km}$ winds early in 2001, and $79 \mathrm{~km}$ winds at the end of that year. Lagged cross correlations were calculated (not shown), and although there were indications of similarity near zero lag, the values were not high. It is evident from the figure that there are differences in spectral content, and that has kept the calculated 60-day correlations rather modest. Time sequences of the zonal winds from the other MFR locations of CUJO demonstrated quite similar behaviour to that of Fig. 1. In contrast, the meridional winds (northward being positive) provided modest differences from Fig. 1, as the amplitudes of the PW responsible for the longer period ( $>2$ days) oscillations are smaller during the winter-like months, while the amplitudes of the summer's 2-d wave are larger (Manson et al., 2003a, 2004a) and are at times dominant. Finally, the lags between oscillations in the lower atmosphere and mesosphere were available from a modest number of selected examples of the correlograms, and proved less noisy than the phases from cross-spectral techniques; the lags were highly variable but are typically a few days.

The wavelets for the daily mean winds from the MFR radars and for TOMS (at the same locations) are shown in Fig. 2. We have placed the highest and lowest latitudes at the top and bottom of the figure, with the data from $40^{\circ}$ latitudes in the middle for best comparisons. Spectra from the MFR radars for the first third (2000-2001) of this observational interval were shown in our earlier paper (Manson et al., 2004a). As in the earlier paper we show only $85-\mathrm{km}$ spectra, as the spectral changes from $79-88 \mathrm{~km}$ (the height range with best data for spectra) are usually quite modest. We have used the same location for the TOMS data as for the MFR for several reasons: we follow Pancheva et al. (2003) at this stage for reasons of comparison; and the low frequency (temporal and spatial) PW are expected to be present over a significant range of latitudes and longitudes near the radar sites (they are often described by global Hough modes). Uncertainties about the latter expectation are the reason for the formation of "regional" harmonic means for wavelets from the 3 radar locations of the CUJO network (North AmericanJapanese $40^{\circ} \mathrm{N}$ locations); this was mentioned in the Analysis Sect. 2.2 and is discussed in Sect. 3.3 below.

Despite the inter-annual variability, which is expected with PWs whose forcings are variable in time and space, the seasonal variability of the oscillations in the MLT winds and TOMS data is consistent with expectations based upon the time sequences of Fig. 1. Generally, and except for Yamagawa $\left(31^{\circ} \mathrm{N}\right)$, the wind oscillations at longer PW periods (12- to 30-d) are stronger in winter-centred months (typically November to March). For the MLT range of altitudes these oscillations are usually associated with the classical westward propagating Rossby "normal" PW modes (the so-called 5-, 10-, and 16-d waves), and the quasi-2-d Rossby-gravity normal mode (Q2D wave) (Luo et al., 2000). This latter wave is at the Nyquist period (2 days) here, but there is spectral energy evident near 2 days in the plots as the bandwidth of this wave is quite broad and aliasing has further spread the spectral peak near 2 days. However, the PW amplitudes at 2-d (summer, NS) and 16-d (winter, EW) are quite comparable at, say Platteville, which is similar to the results of the specific analysis of these two waves (Manson et al., 2004a). The ranges of observed periods for these PWs are typically 2, 5-7, 8-10 and 12-22 days due to Doppler shifting by the background winds (Luo et al., 2002a, b). There is also spectral energy evident in Fig. 2 for those same winter months at longer periods (circa 22- to 30-d) associated with "extra long periods" that have correlations with the solar rotation period (Luo et al., 2001). For the remaining months the MLT wind activity is usually restricted to periods of less than 12 days. The color scale (range of $\mathrm{dB}$ with respect to color) was 
Daily Mean Wind vs. TOMS (DU)

NS
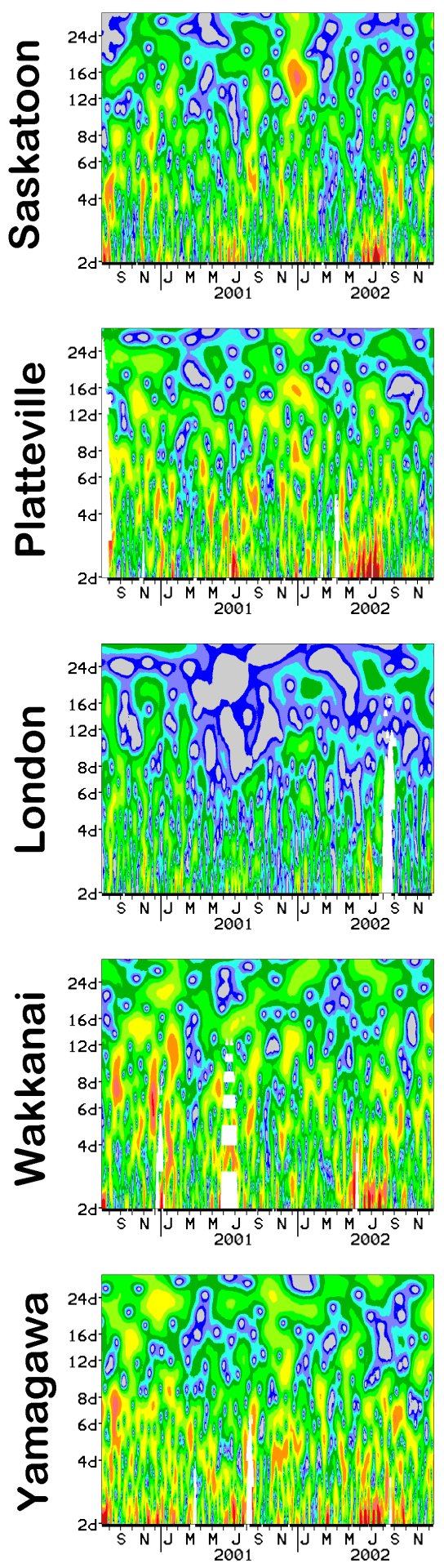

$85 \mathrm{~km}$
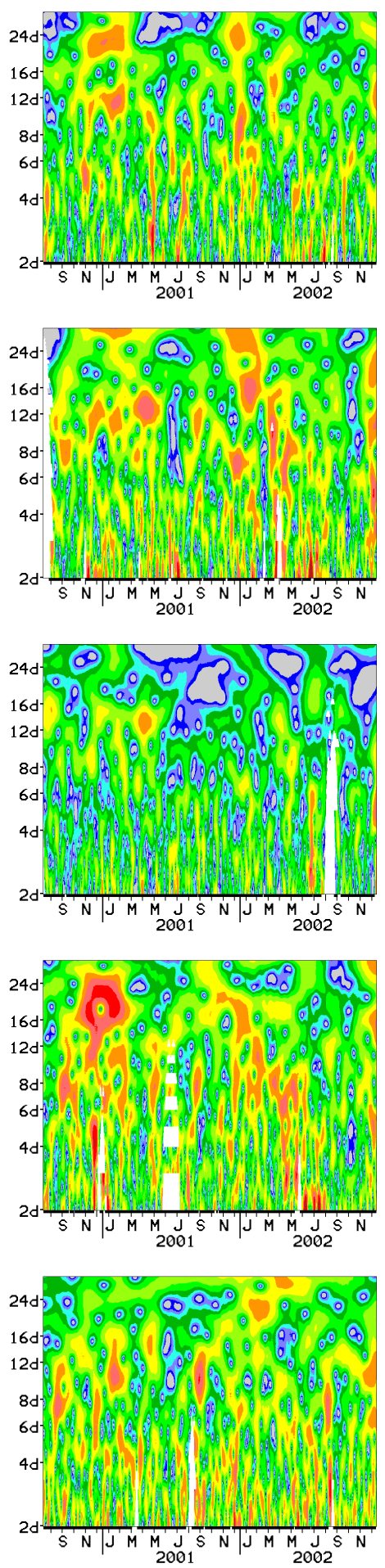

$-220 \log _{10}(\mathrm{~m} / \mathrm{s})^{28}$
OZONE
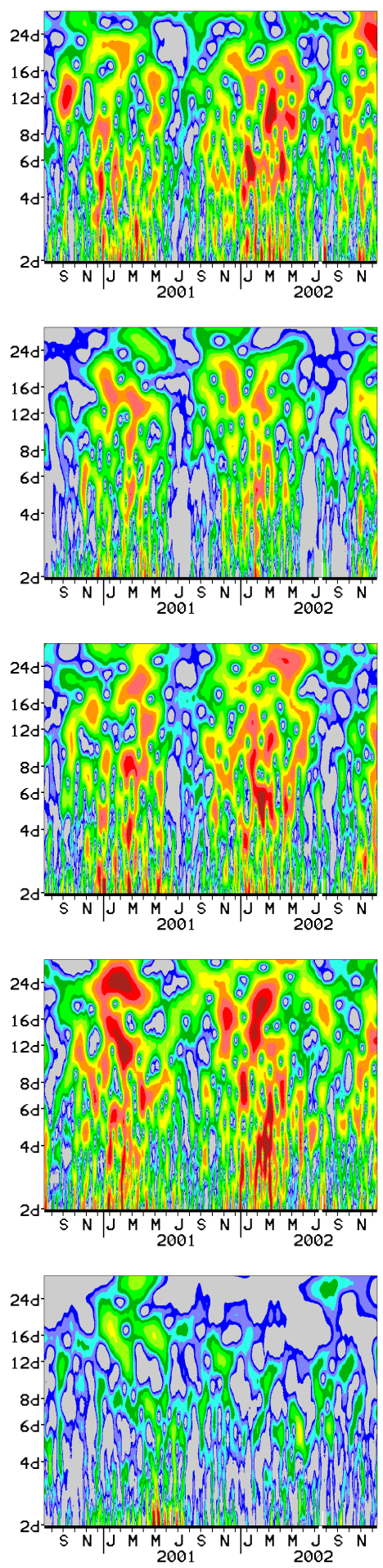

$1020 \log _{10}(\mathrm{DU}) 30$

Fig. 2. Wavelet spectral plots for the $85 \mathrm{~km}$ daily mean winds (zonal EW, meridional NS) and the ozone (DU) values of TOMS for the radar locations of the CUJO network. 
chosen to allow for a best comparison of the various MFR wavelets. These do differ significantly between, say, London and Wakkanai. We add, in revision, that the very detailed assessment of the wind analysis used at London is revealing that a factor of typically $30 \%$ (smaller winds) is involved. This correction would change yellow contours in Fig. 2 to orange $(2 \mathrm{~dB})$ and bring the wavelets into closer agreement with those for Platteville. The work is in progress and so no changes are made to the figure.

The TOMS oscillations, while also maximizing in the winter months, do have an additional temporal different character; and here some care is required by the reader, as the color scale differs a little from that of the MLT winds, and was chosen to optimize the TOMS regional differences. However, the TOMS characteristics are unique: the interval of enhanced broad-spectral activity (yellow-red) is longer in duration and extends from November to April; and the activity in the remaining months is less at all periods/frequencies (whereas for the MLT winds, although spectral changes occur, there are peaks of similar intensity in the summer and winter months). It is interesting that the variation in intensity of ozone oscillations between sites also often differs from that of the MLT winds: although TOMS for the Wakkanai location also has higher intensities, values at the London site are not low (they are similar to those at Platteville), and activity at the Yamagawa site is very low.

It is appropriate to comment further on the relationship between spectral variability of the TOMS data (measured in DU) and PW activity. The considerations of Schoeberl and Krueger (1983) have already been mentioned in the Introduction, where it was noted that they showed that evanescent waves (including baroclinic waves) are most effective in providing an ozone perturbation. More recently, Fusco and Salby (1999) demonstrated that "variations of up-welling wave activity" (as determined by the Eliassen-Palm flux) correlate very highly (99\% level) with the increases in total ozone. The variations in PW activity "modulate ozone transport and chemical production by the diabatic mean circulation" and "account for much of the variability in measurements of total ozone". The PWs involved have the greatest activity in the winter months, and include the baroclinic waves of the tropospheric weather systems, which are largely absorbed in the lower stratosphere (Charney and Drazin, 1961). The scale sizes of these waves are of small to medium size (typically more than zonal wave number 4 ), leading to small so-called "critical speeds" (which vary inversely as the square of the wave numbers) in their formulation (see also Andrews et al. (1987) for a very nice presentation, page 178); even if the phase velocities (c) are relatively modest and westward relative to the eastward winter flows (u), the (relative) intrinsic phase velocities ( $\mathrm{u}-\mathrm{c}$, Doppler shifted) will often easily exceed the "critical speed", which is then the condition for negative squared refractive index. This in turn leads to wave dissipation and strong damping. Thus, many of the smaller scale PWs effective in providing spectral peaks in the TOMS wavelets are likely to be often different in characteristic from those (westward propagating, large-scale normal modes) dominating the MLT wavelets.

Our studies of PW, in particular the 16-d wave, have shown that there is considerable intermittency in the wave activity, and a lack of strong correlation in the occurrence of bursts of activity between radar sites (Luo et al., 2002a, b). Thus, only oscillations in that band (12- to 22-d) which are strongly evident at several locations (simultaneous bursts of activity involving several complete oscillations) provide phase differences consistent with coherent westward propagation and unambiguous determination of wave number $(\mathrm{m}=1)$ (Luo et al., 2002b). Examples of such bursts are the circa 12- to 16-d PW (EW) during April 2001, and spectral activity near 16 days in January-February of 2001 and 2002; there are also several spectral peaks in the TOMS data at these times. Consistent with that notion we will later show the harmonic means of the 3 pairs of wavelets (London, Platteville and Wakkanai) for the daily mean winds from Fig. 2, and also harmonic means including the TOMS wavelets. This analysis was used by Manson et al. (2004a). Those later figures will also include the harmonic-mean wavelets for the tidal data, so that wind variability (directly related to $\mathrm{PW}$ ) and tidal variability (e.g. due to changes in tidal forcing at PW periods, or PW-tide interactions) can be compared. However, we will first show the wavelets appropriate to the individual sites for the MLT tides, accompanied by only modest comment.

\subsection{Variability of Total Ozone and Mesospheric Tides at CUJO Sites}

Time sequences of the daily mean tides (12-, 24-h), in comparison with the TOMS daily samples, were examined for each location. It was clear from these and from correlations over 60-day intervals that the linkages were not strong. Complex spectral structures for the two types of data sequences were evident. The wavelets for the NS and EW components of the respective tides at $85 \mathrm{~km}$ for the 5 MFR locations are shown in Figs. 3 and 4, along with the same TOMS wavelets as before (Fig. 2). Variations in the tidal amplitudes at PW periods can be caused by at least two different processes: the temporal variation in the ozone associated with PW activity in the stratosphere can provide a temporal variation in the forcing of the tides, which will lead to variations or oscillations in the local MLT tidal amplitudes at those same PW periods; or PWs, which have a signal within TOMS data, may propagate into the MLT where they non-linearly interact with the tides to produce a beating or oscillation of the tidal amplitudes, again at the tidal period. Pancheva et al. (2003) mention other mechanisms (e.g. non linear interactions in the stratosphere), and suggest that longer time lags may be associated with processes involving propagation of PWs into the mesosphere. Planetary scale oscillations in the background winds of the stratosphere and mesosphere will also cause significant variations in the tidal amplitudes (Jacobi et al., 2001). Such processes lead to longitudinal changes in tidal characteristics, which may make it more difficult to discern other nonlinear PW-tidal interactions e.g. lower signal-tonoise levels when the tidal amplitudes are small locally. 


\section{Semi-Diurnal Tide Amp. vs. TOMS (DU)}

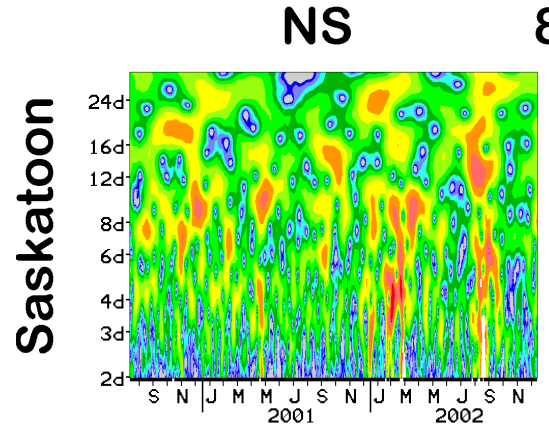

\section{$85 \mathrm{~km}$}

EW
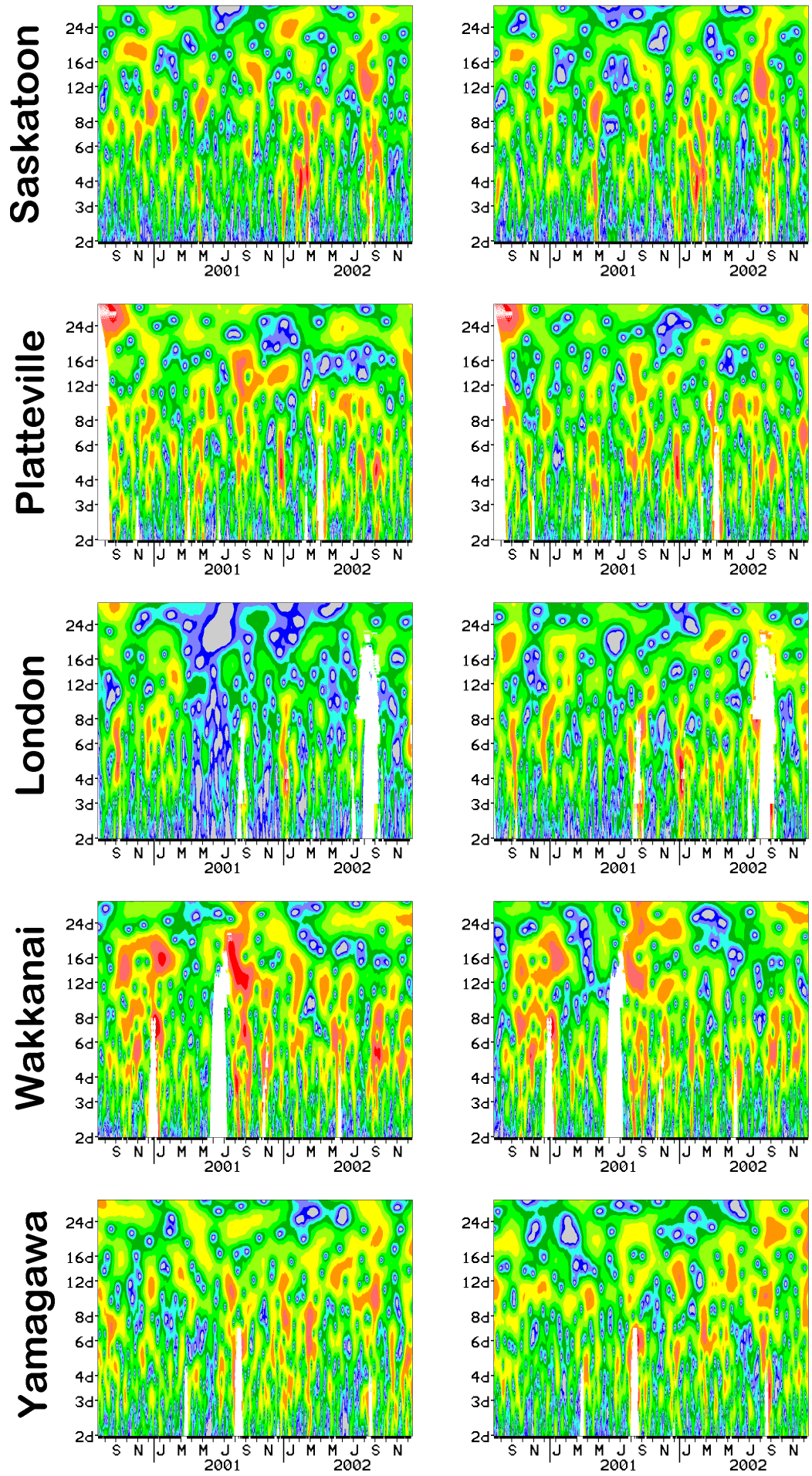
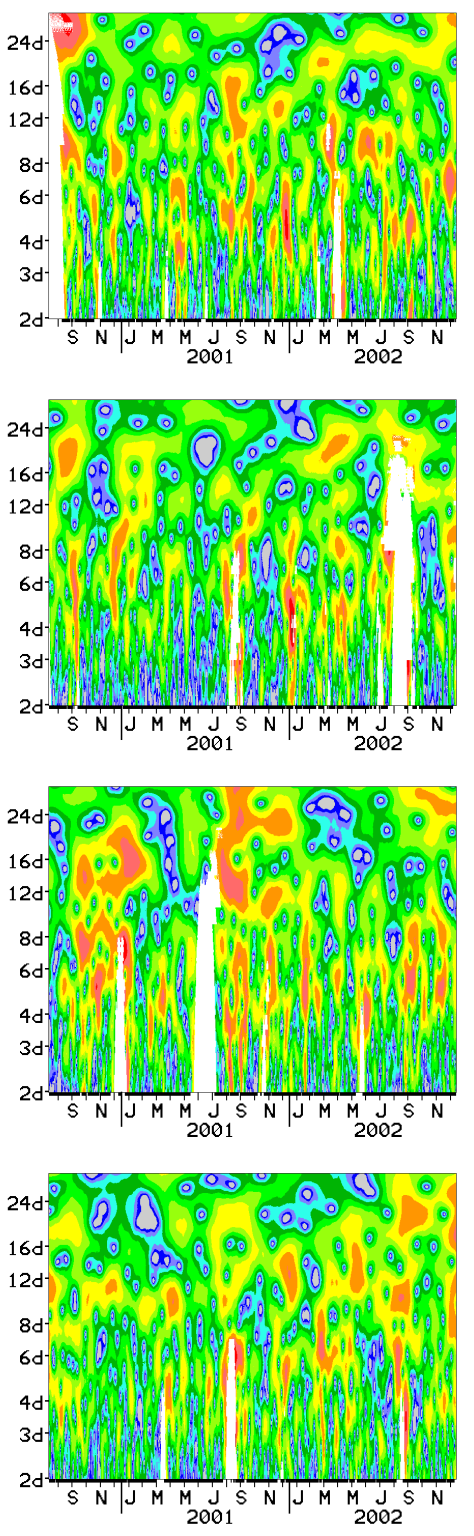

OZONE
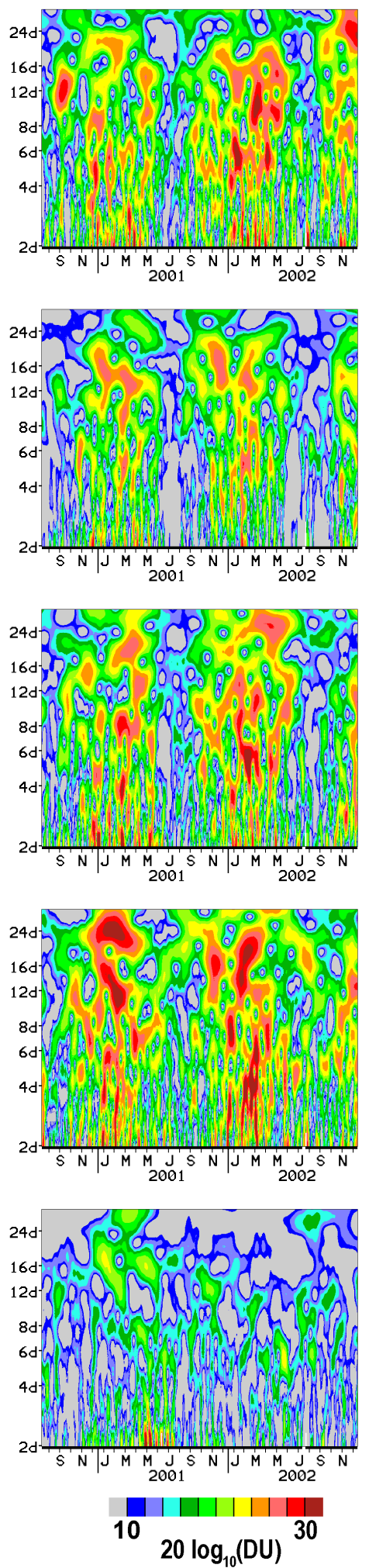

Fig. 3. Wavelet spectral plots for the $85 \mathrm{~km}$ daily $12-\mathrm{h}$ (semidiurnal) tidal amplitudes (zonal EW, meridional NS) and the ozone (DU) values of TOMS for the radar locations of CUJO network. 


\section{Diurnal Tide Amp. vs. TOMS (DU)}

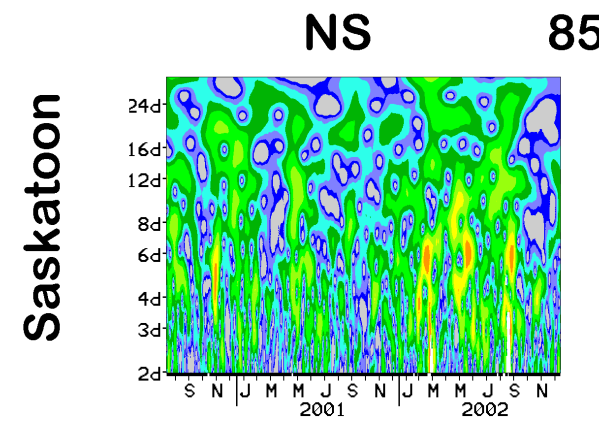
$85 \mathrm{~km} \quad \mathrm{EW}$ OZONE
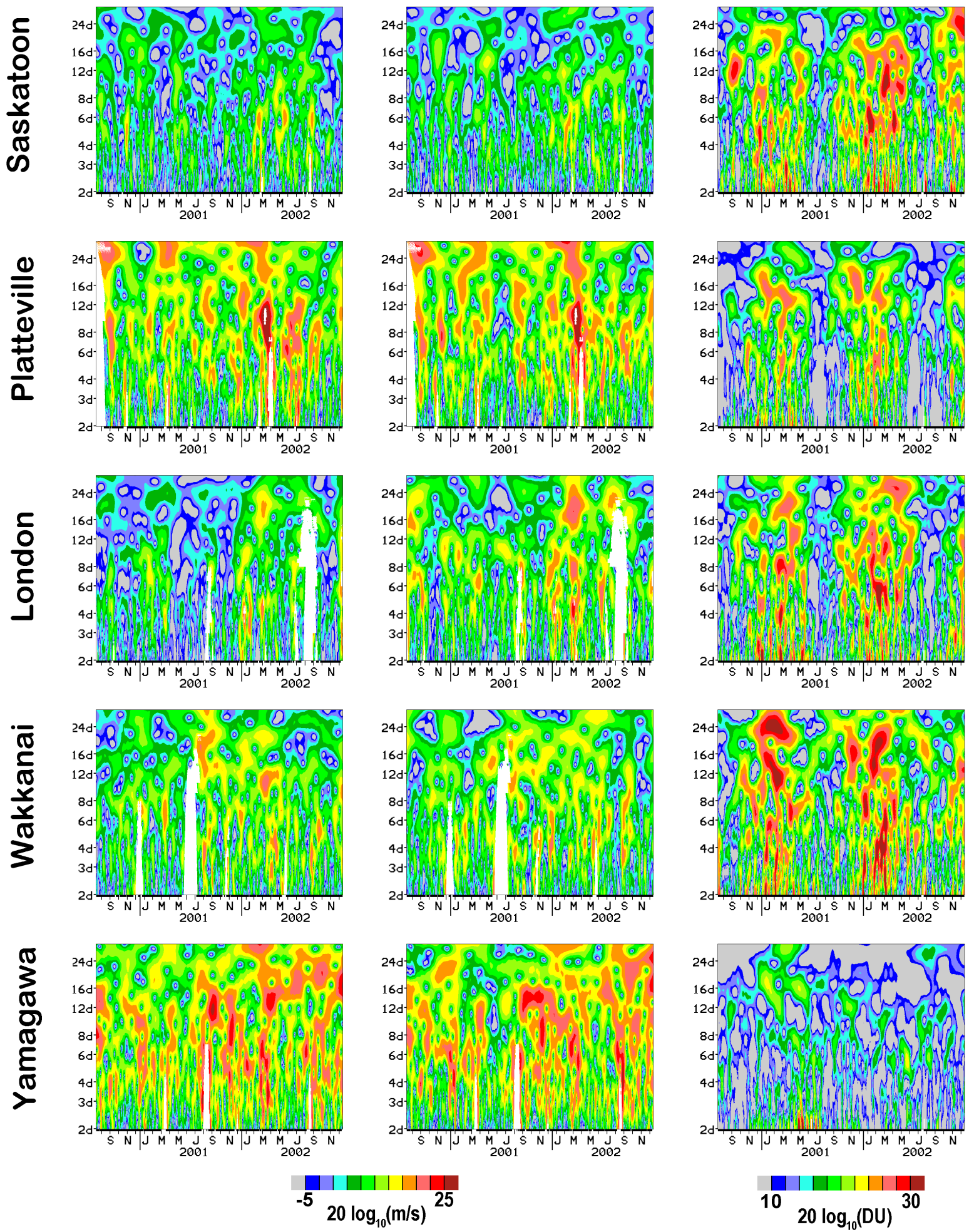

Fig. 4. Wavelet spectral plots for the $85 \mathrm{~km}$ daily 24-h (diurnal) tidal amplitudes (zonal EW, meridional NS) and the ozone (DU) values of TOMS for the radar locations of CUJO network. 
Considering the 12-h tide firstly (Fig. 3), the EW and NS components are very similar for each location. This is consistent with the circular nature of the tide and similarities in the amplitudes of the two tidal components (Manson et al., 2003a). Seasonal variations in the tidal oscillations are somewhat less clear than for the mean winds of Fig. 2, although again the summer months are more likely to have less activity and shorter periods. Despite the intermittency, there are temporal and spectral similarities between the three $40^{\circ} \mathrm{N}$ MFR sites e.g. an 8-d burst during December 2000, and a 12- to 16-d burst in early September 2001. There is significant spectral energy in the TOMS wavelets for the former event, so coupling could be involved. Otherwise, there is activity at periods near 16 days during the middle of both winters. There is also an indication that the oscillations in the tides are larger in the winter, and the autumn, when the amplitudes of the 12-h tide are themselves largest (Manson et al., 2003a). This may well favour the nonlinear PW-tidal process, as larger tides will lead to larger oscillations.

Finally the wavelets for the 24-h tide (Fig. 4) are interesting, in that the coupling of this tide with the stratospheric ozone and its variability has not been considered before. However, the forcing of this tide by stratospheric ozone is significant (Hagan, 1996), although it has often been ignored by some in discussions of this type. The differences in wavelet intensities between the $40^{\circ} \mathrm{N}$ locations, and between them and the $52^{\circ} \mathrm{N}$ and $31^{\circ} \mathrm{N}$ sites, is quite considerable, and reflects to some degree the decreasing amplitude of this tide with increasing latitude. An example of a common burst of MLT activity is at 16-d near March 2002, for both the EW and NS components; there is again spectral energy present in the TOMS spectra at this time, so that coupling may exist. The variation in seasonal activity of the 24-h tide is not strong. This suggests that much of the MLT variability in summer-centred months is not related to local TOMS (PW and ozone) variability.

\subsection{PW activity and coupling over the CUJO region}

As we discussed in Sect. 3.1 it is desirable to form geometric means of the three pairs of wavelets appropriate to the $40^{\circ} \mathrm{N}$ locations for the mean daily winds and for both tides. This process effectively locates in time and spectral space the MLT events that are simultaneous and most likely to be coherent in the Pacific-North American sector sampled by CUJO. We show in Fig. 5 the mean MFR wavelets (specifically geometric means of the MFR wavelet amplitudes from the three $40^{\circ} \mathrm{N}$ locations are used), and in Fig. 6 we include the TOMS amplitudes in the mean wavelets. The latter should locate the bursts or events that are common between the lower atmosphere (circa $100 \mathrm{mb}$ ) and mesosphere (or MLT) over this large region of the planet, and which should involve coupling of some type. Common $\mathrm{dB}$ values and colors are used to allow for comparisons of components and of wave type. For comparison with previous studies, the paper by Pancheva et al. (2003) involved the 12-h tide for Sheffield $\left(52^{\circ} \mathrm{N}\right)$ and a presentation similar to that of the MLT tidal-
TOMS data of Fig. 6; while the paper by Lawrence and Jarvis (2003) provided information on the winds and could be compared with the appropriate (lower) sections of Figs. 5 and 6.

We consider firstly the mean winds of Fig. 5. The wavelets for the mean or background winds provide a clearer summary of the seasonal variations discussed in Sect. 3.1. The activity is dominant in the EW component, for periods longer than about 8 days, which is consistent with the polarizations of the "normal" waves at mid-latitudes; and winter-centred activity is dominant with the longest periods occurring in mid-winter. Although the spring activity is limited spectrally, the intensities remain large. There is considerable activity in the 16-d range (12-22 days), the 10-d and even in the Q2D ranges throughout the winter-centred interval. Bursts which are orange-red in color (above $14 \mathrm{~dB}$ ) have significance levels in the individual wavelets (Figs. 1-3) in the 95-99\% range (see Sect. 2.2 for discussion of significances). Interannual variability is evident and expected with such oscillations, which are responding to geophysical "noise" and variability in the system for their sources.

The products of the wavelets for TOMS ozone and the MLT winds (Fig. 6) provide indications of coupling: the summer-winter differences are enhanced due to the minimal summer PW activity in the TOMS wavelets; and substantial bursts of activity are in common between the two atmospheric regions, especially from December to May. Although some of the PW-related variability in the TOMS data has not impacted the mesosphere (there was discussion in Sect. 3.1 on the PW of the lower atmosphere), it appears that much of the PW flux detected by the TOMS systems has propagated directly into the mesosphere. Careful inspection also demonstrates that it is for the bursts of activity greater than 10 days in period that the similarities between the mean wavelets of the MLT winds (Fig. 5) and MLT winds-TOMS products (Figs. 6) are the greatest. Features which were noted earlier from perusal of Fig. 2 are very clear in both Figs. 5 and 6; in particular the aforementioned 12- to 16-d (EW) event toward the end of April 2001, and the 16-d events in JanuaryFebruary of 2001 and 2002. (These features are clearly evident in the harmonic-mean TOMS wavelet (not shown).) For the winters of 2000/2001 and 2001/2002 there were also stratospheric warming (SSW) events (U.S. National Weather Service, NCEP (National Centres for Environmental Prediction) data) which in both cases involved December pulses at high and polar latitudes followed by events in February. There was substantial MLT and stratospheric PW activity in both years near these times (Figs. 5, 6) but specific causality is difficult to establish. Jacobi et al. (2003), meanwhile, noted the presence of an intensifying PW of a 10 day period at the time of the SSW of 2000/2001 in the westernEuropean and Scandinavian sector. Returning to the individual MFR wavelets of Fig. 2, there is activity near 12 days during February at Saskatoon, the most northern location; and there is also a feature in the regional CUJO wavelet (Fig. 5) at this time. This result is encouraging, but a special independent study would be needed to verify the details, and this is an activity for the future. 


\section{Geometric Mean Tidal Amp. \& Background: $\sqrt[3]{\text { PxLxW }}$}

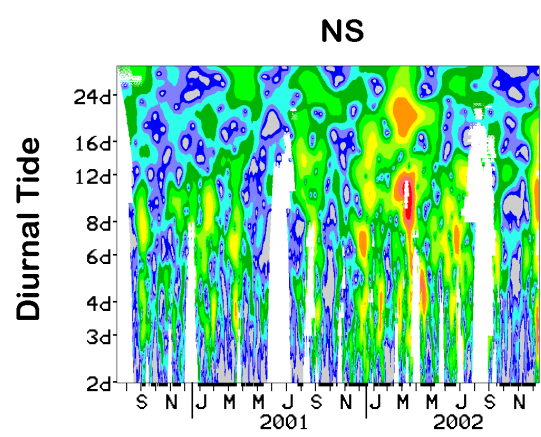

$85 \mathrm{~km} \quad \mathrm{EW}$
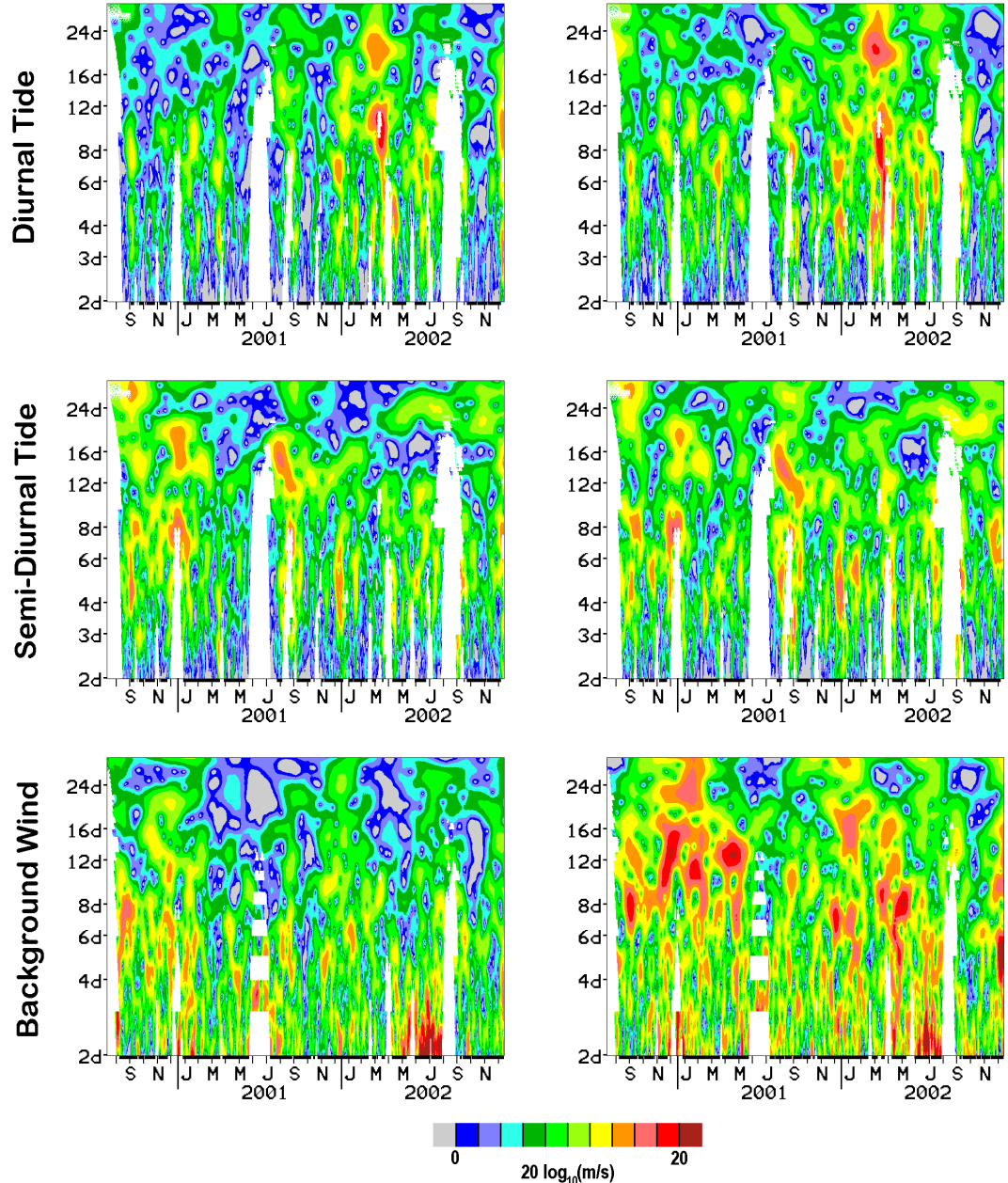

Fig. 5. Wavelet spectral plots for the $85 \mathrm{~km}$ daily winds, 12-h (semidiurnal) and 24-h (diurnal) tidal amplitudes (zonal EW, meridional NS). These plots are geometric means of the individual wavelets for the three $40-45^{\circ} \mathrm{N}$ MF radars (London, Platteville, and Wakkanai) of the CUJO network. A gap is shown if an amplitude value is unavailable for one or more sites.

Pancheva et al. (2003) noted several regular features during the four years 1989-1993: 16-d PW bursts were implicated (the oscillations were in the 12-h tide) in the autumn (September-October) for each of the two years having easterly phases of the QBO (negative westward stratospheric flow), and stronger 27-d oscillations were noted during winter for the other westerly QBO phases. In Figs. 5 and 6 there is a much less clear morphology for the 16-d oscillation, based upon the National Weather Service (Climate Prediction Service) analysis, whose QBO $30 \mathrm{hPa}$ index indicated that easterly flow dominated the tropical latitude circles for the last 6 months of 2000, and all of 2001 . The flow was westerly during 2002. In the mesosphere (Fig. 5) the 16-d PW winds have a modest autumn burst and a stronger long period (circa 24-d) burst during the 2000/2001 months (September-April) of easterly QBO phase (these features are less evident in the TOMS/winds products of Fig. 6).
However, there was no 16-d burst for the autumn of 2001 when the flow was still easterly (the QBO was in the midst of a phase transition from the short-lived biennial oscillation). It is not surprising to see this difference for the 16-d PW during these two different intervals (1989-1993 and 2000-2002). Luo et al. (2000) assessed the 16-d PW activity at Saskatoon for 1980-1996 in comparison with the QBO phases, and found complex and often weak correlations. Some months exhibited weak correlations with the westerly phase, and others the easterly phase.

The phase differences or lags between stratospheric and mesospheric PW activity are best assessed through 60-d correlograms previously mentioned in Sect. 3.1. However, the phase differences vary from site to site, and with the interval chosen, as well as with the period dominating the correlograms. A broad generalization, based upon these correlations and also phase differences from the complex wavelets, is that 


\section{Geometric Mean : $\sqrt[4]{\text { DUXPXLxW }}$}
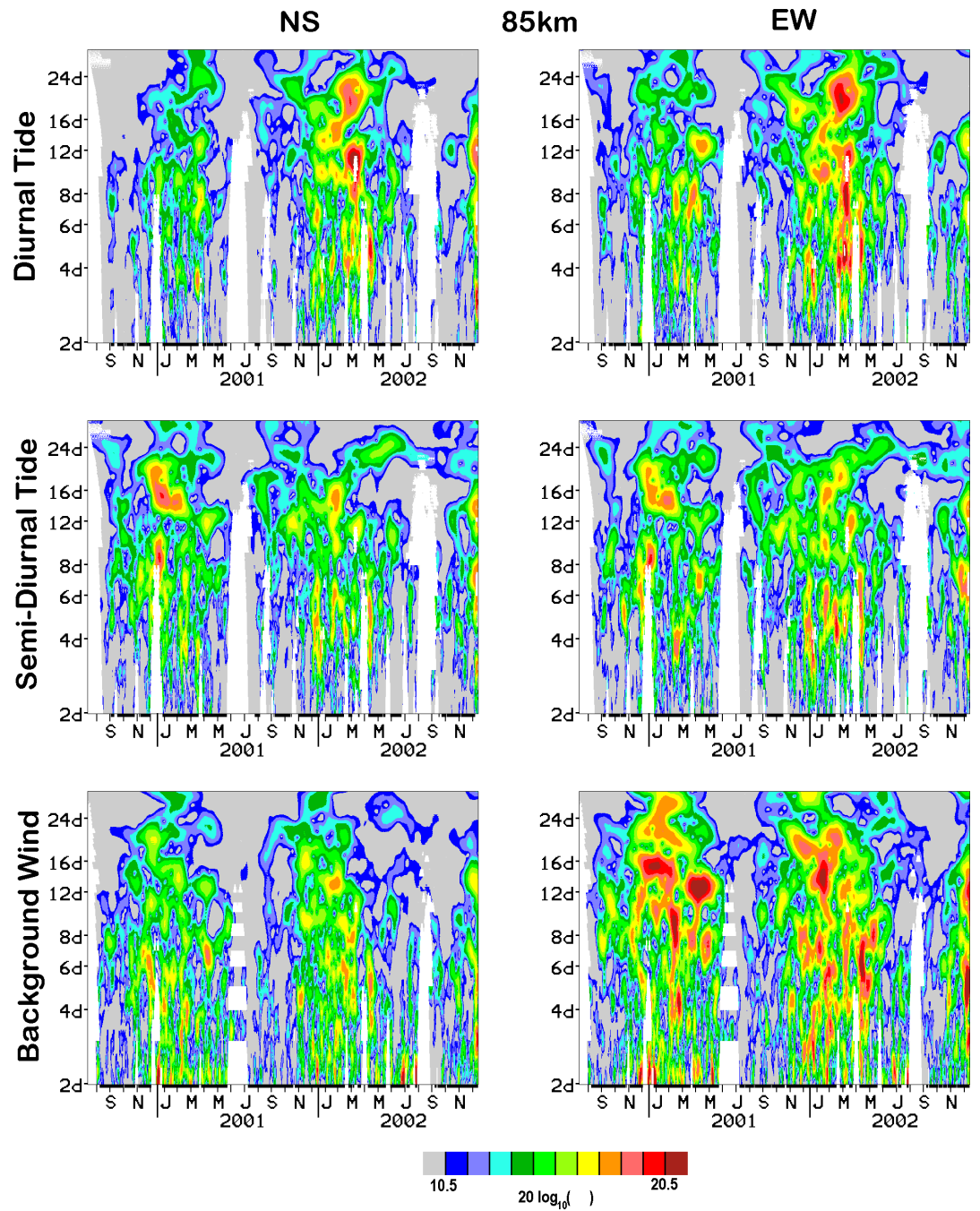

Fig. 6. Wavelet spectral plots: geometric means of the wavelets for the $85 \mathrm{~km}$ daily winds, 12 -h (semidiurnal) and 24-h (diurnal) tidal amplitudes (zonal EW, meridional NS) shown in Fig. 5 and the wavelets for the ozone (DU) values of TOMS for the same locations. The log is of the value of the term in the heading of the figure.

the MFR-derived phases for the longest periods often differ from the TOMS phases by only a few (+/-2) days. It is in fact not obvious what phases are to be expected. As noted by Schoeberl and Krueger (1983) the phase differences between the vertical and horizontal perturbation velocities and the maxima of ozone in the stratospheric-tropospheric PW depend on the period of the wave; in addition, the propagation conditions and the vertical wavelength of the PW will affect the final phase difference between the TOMS oscillation of interest and the mesospheric PW.

The patterns of activity at PW periods for the tidal amplitudes in Fig. 5 are quite different from the mean winds. The general levels (in $\mathrm{dB}$ ) are lower, with the 12-h tide showing modest bursts (orange and red; $>14 \mathrm{~dB}$ ) that are also more limited temporally in the autumn and winter, and the 24-h having winter and spring bursts. The processes involved in tidal variability are potentially more complex and numerous than those for the background wind, so that identical activity in all wavelets of Figs. 5 (and 6) are not to be expected. For the 12-h tide there are several 16-d bursts (in particular the previously noted event of early September 2001, as well as mid-winter events during both winters, in Fig. 3), 8-d bursts (that of December 2000 was seen in Fig. 3), and frequent 4- to 6-d activity. The cross-product with the three TOMS wavelets (Fig. 6) again provides a stronger seasonal trend, and accentuates some different bursts e.g. the September 2001 burst is missing. However, the 8- and 16-d bursts of December-January 2000-2001, as well as the 16-d event in the mid-winter of 2002, are again evident. There is excellent agreement between the NS and EW wavelets, which is encouraging; the tidal components are not completely independent in the analysis, but the strong similarities provides 
a robust indication of PW activity in common between the lower atmosphere and mesosphere. As discussed previously (Sect. 3.2) the physical processes providing modulation of the tides do differ, at least in detail, from the PW signal in the winds; however, some of the features in the MLT tidal-TOMS wavelets coincide with features in the MLT (EW) wind-TOMS wavelet e.g. the 16-d features of JanuaryFebruary of 2001 and 2002, indicating the involvement of a common PW in both.

Finally, the 24-h tidal wavelets demonstrate coupling between the lower and middle atmosphere (Fig. 6), and somewhat greater inter-annual variability than for the 12-h tide (Fig. 5). The bursts of activity in the MLT tide-TOMS wavelets (Fig. 6) often occur at similar temporal-spectral locations to that for the 12-h tide e.g. the 16-d burst of JanuaryMarch 2002; but there are also striking differences e.g. the 24-h tidal activity during 2002 is clearly stronger than in 2001. Again, given that the 24-h tide's mean wavelets for the mesosphere over London-Platteville-Wakkanai have winterspring maxima, closer in morphology to the TOMS variability, the MLT tide-TOMS products of Fig. 6 are very similar in temporal-spectral features to those for the mesosphere (Fig. 5).

The presence of the apparent coupling between the 24-h tide of the MLT and the TOMS parameter, which has both PW and ozone variability associated with it, is interesting and perhaps not expected as much as for the 12-h tide. This may suggest that the MLT spectral variability involves the interactions of PW and tides (in stratosphere or mesosphere) rather than the simple radiational forcing of the tide by variable ozone concentrations, given that processes involving water vapour are normally considered to dominate the forcing of the 24-h tide in the lower atmosphere. However, as noted from the modelling experiments of Hagan (1996), the role of stratospheric ozone in the 24-h tide's MLT amplitudes is substantial. The lags between stratospheric and mesospheric activity are again available, in principle, from the correlograms or complex wavelets. However, the assessments are again not simple, with phase differences varying with time interval, year and dominant period. Lags of several days are typical.

\section{Wave numbers of PW in the stratosphere and meso- sphere and discussion of coupling processes}

There has been considerable discussion of the character of PW in the lower atmosphere/stratosphere and mesosphere/MLT already in this paper. We noted in the Introduction that Fedulina et al. (2002) had shown that at $1 \mathrm{hPa}$ in the NH there was considerable spectral intensity for both the eastward and westward propagating PW, with the westward PW of largest scale $(\mathrm{m}=1)$ dominating; and that Schoeberl and Krueger (1983) identified evanescent PW of synoptic scale (including the baroclinic waves), which are mainly eastward propagating, as being most effective in producing the maximum ozone signal in the column values. Some earlier studies include the seminal paper by Venne and Stanford (1982); they noted spectral energy for eastward and westward propagating waves of long period (above 5 days) in both hemispheres; however, the analysis of NH data was hindered by poorer Nimbus 4 data (Nimbus 5 existed), significances were low, and unlike the $\mathrm{SH}$, no clear predominance of eastward motions was evident. Their results are, however, qualitatively similar to Fedulina et al. (2002).

In contrast, the PW of the MLT most frequently studied, and discussed here in Sect. 3.1, have been the Rossby or "normal" modes. Relatively well-behaved westward propagation has been demonstrated by the 16-d PW (e.g. Luo et al., 2002b), for large simultaneous bursts of activity, and by the quasi 2-d Rossby-gravity normal mode (e.g. Thayaparan et al., 1997). We have also argued in Sect. 3.1 that the smaller scale (typically wave number m greater than 4) synoptic waves of the troposphere and lower stratosphere will be largely absorbed (due to their relatively small "critical speeds", or eastward motion relative to the background wind).

Nevertheless, the products of the wavelets for the TOMS ozone and MLT winds in Fig. 6 showed considerable common activity in winter-centred months (temporally and spectrally), suggesting that significant portions of the PW flux detected by the TOMS systems has propagated directly into the mesosphere. It is the bursts of TOMS activity with periods greater than 5 days that have the greatest similarity to the MLT activity in the daily mean winds (Fig. 5). It is worth noting that the largest PW $(m=1,2)$, some of which may have eastward phase speeds (c) at the TOMS-data heights, and which may also be moving westward relative to the comparatively strong winter eastward flows $(\mathrm{u})$ of the stratosphere and mesosphere, appear to meet the conditions for propagation into the middle atmosphere. These conditions are that the intrinsic (relative-zonal) phase velocities $(\mathrm{u}-\mathrm{c})$ are positive, and also smaller than the relatively large "critical speeds" for these large-scale waves (Charney and Drazin, 1961; Andrews et al., 1987). We discussed this formulation briefly in Sect. 3.1. Such waves could be associated with jet stream movement and vortex variability, and have not been recently considered as major contributors to MLT dynamics. They appear to be worthy of renewed study with models (Pogoreltsev, private communication, 2003). These waves should propagate into the middle atmosphere more easily than the stationary PW and certainly more easily than the westward moving (relative to the ground) Rossby PW of similar scale, since their intrinsic speeds are smaller. It is possible, as suggested by a reviewer, that these waves may propagate only to the stratopause; however they may become evanescent above there and reach into the mesosphere and therefore be detected with the MLT radars. (The Charney and Drazin (1961) formulation for propagation works qualitatively well for Rossby PW such as the 16-d wave: despite the intrinsic phase speeds being larger than for the stationary and largest scale eastward propagating PW, they are still usually smaller than the "critical speeds" in the NH winter months. This is consistent with the climatologies shown in 


\section{TOMS Wavenumbers 2001}

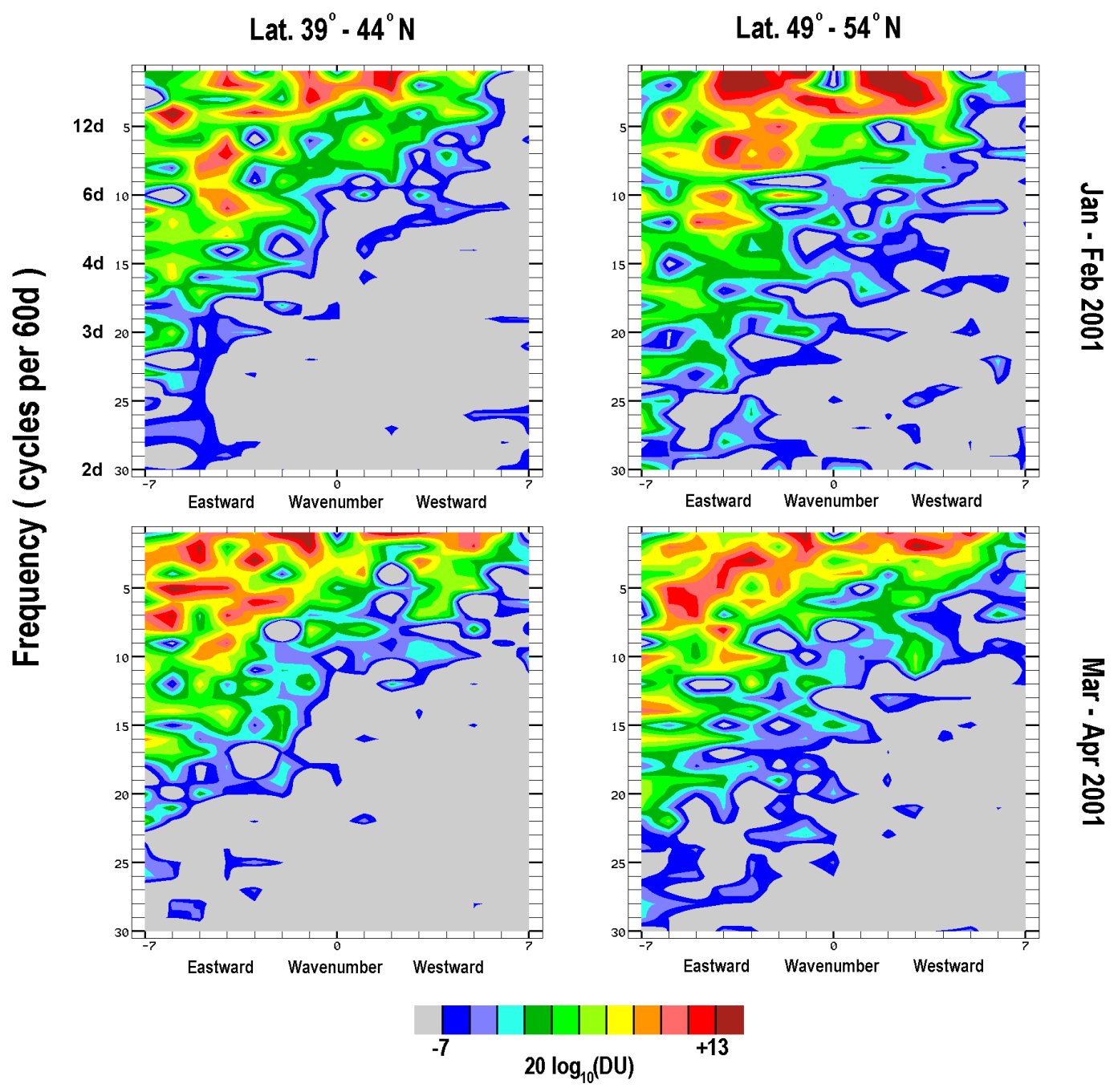

Fig. 7. TOMS wave number spectra for two latitude bands during the days $1-60$ of 2001 . The full set of available longitudes is used for these spectra.

Figs. 2, 5 and 6. However, as discussed by Luo et al. (2002a), using modelling results in the $\mathrm{SH}$, the winter eastward MLT winds are stronger, the intrinsic phase speeds larger, and propagation is discouraged as the appropriate "critical speed" is exceeded.)

As a further diagnostic of the PW activity, wave-number analysis has been described in Sect. 2 and applied to the TOMS data. This will provide insights into the scales and direction of propagation of the PW at the $100 \mathrm{mb}$ (circa $20 \mathrm{~km})$ level, and hence the processes for coupling with the MLT. This follows our most recent work (Manson et al., 2004a), where the analysis of multiple-site radar data to obtain wave numbers for PW-scale oscillations in the wind field was provided for the year 2000/2001. While for periods greater than 7 days the preferred wave number was one $(\mathrm{m}=1$, westward), consistent with the frequently observed 16- and 10-d PW, there was considerable spectral energy at $\mathrm{m}=3,4$ and -2 from October to April. Due to the limited number and location of the radar sites, aliasing was possible, and it was argued there that statistical coupling of mode-pairs whose wave numbers differed by $+/-3$ may have affected the process. Thus numbers $m=4$ and -2 could have been appropriate to an actual $m=1$. However, the originally calculated values could well be correct descriptors of the PW. The data in this recent study were of the highest quality available, and indeed are for the year 2000/2001 which is also used here for the wave number analysis.

Wave number analysis of the TOMS data is now shown in Figs. 7 and 8. Days 1-60, and 61-120 from each of the years 2001 and 2002 have been chosen, with latitude ranges of 39$44^{\circ} \mathrm{N}$ to cover the CUJO network of three $40^{\circ} \mathrm{N}$ MFRs, and $49-54^{\circ} \mathrm{N}$ to include the Saskatoon MFR but also to provide a range of latitudes for assessment of spatial variability. As described in Sect. 2, the spectra are coherently averaged over 


\section{TOMS Wavenumbers 2002}

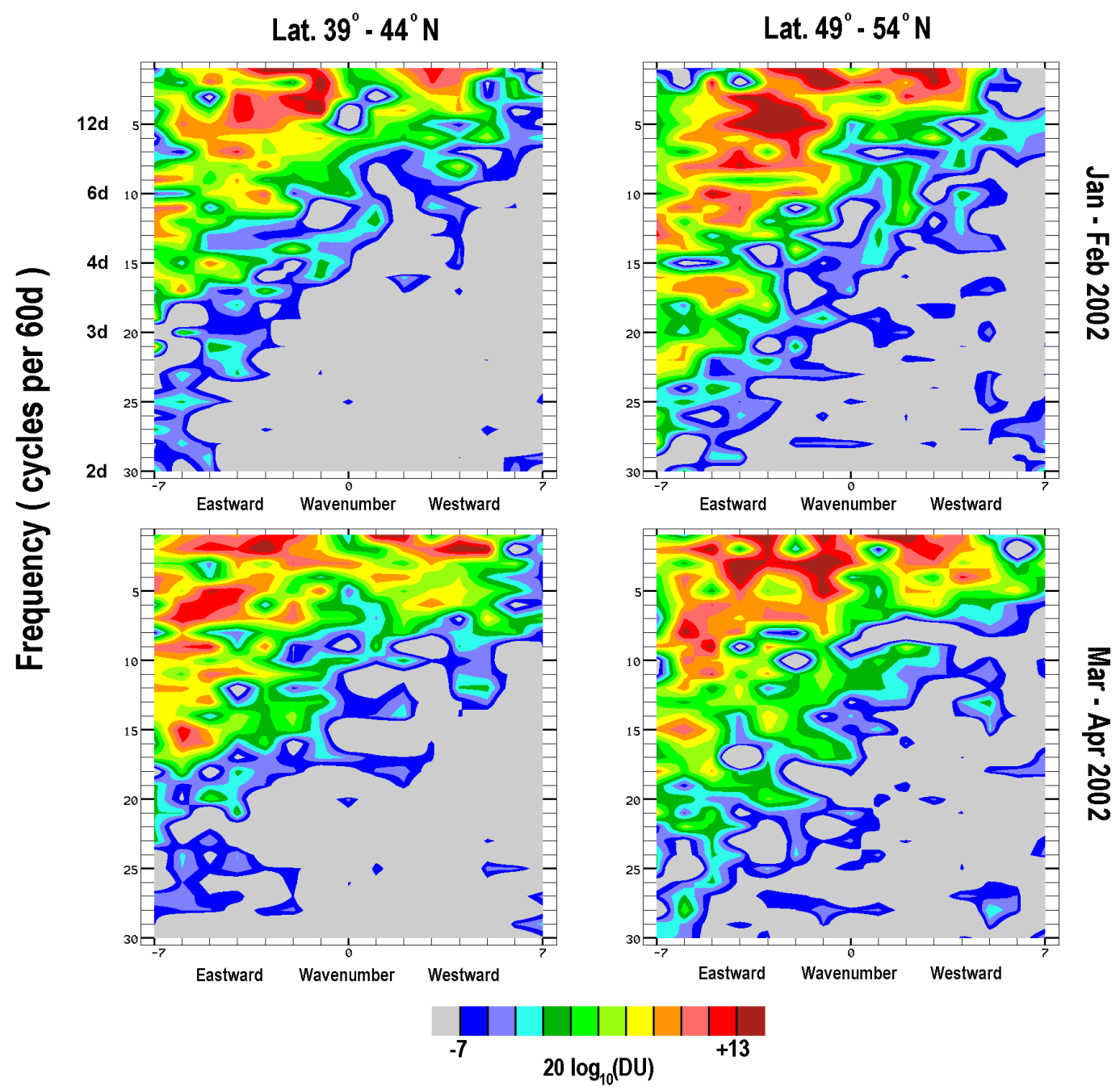

Fig. 8. TOMS wave number spectra for two latitude bands during the days $61-120$ of 2001 . The full set of available longitudes is used for these spectra.

these latitude bands before wave number analysis is applied, so that the intensity contours in these figures represent coherent averages. Based upon the analysis of global HRDI data (UARS, Upper Atmosphere Research Satellite) for the MLT winds, the results of which are contained within Luo et al. (2002a), strong variations of PW activity with latitude (even those oscillations having coherence at all longitudes) are a common occurrence. Hence, variations in spectral features between $39-44^{\circ} \mathrm{N}$ and $49-54^{\circ} \mathrm{N}$ in our figures are expected, and are indeed evident.

The general trends in these plots of the winter and spring PW activity are for dominant eastward propagation of spectral components $m=-1$ to -5 for periods of typically 5 to 30 days (there is also spectral energy at the lowest frequency appropriate to periods near 60 days that would be associated with seasonal transitions). The westward components are more restricted to periods of 12 days and more, although the intensities of those are comparable to the eastward components. It has just been argued above, that eastward PW of large scale and long period ( $>10-\mathrm{d}$ or so) should be able to propagate into the stratosphere and perhaps mesosphere, along with the westward PW, providing that their intrinsic phase velocities are less than the "critical speed" (Charney and Drazin, 1961; Luo et al., 2002a). Indeed, there are high spectral intensities (red contours) in Fig. 7 that often include the PW wave numbers featured for the CUJO radar wind data (1, 3, 4 and -2; Manson et al., 2004a) for this same winter (2000/2001), although other wave numbers have comparable intensities. Of course, all waves at $100 \mathrm{mb}$ are not expected to reach the MLT; and the CUJO network is not global, while the global data set was required for the TOMS wave number analysis. Extended radar coverage is desirable. Finally, there is variability of the wave number contours of Figs. 7 and 8 with latitude (consistent with the HRDI analysis of the MLT 
activity), and also with interval (1-60, 61-120 days of 2001 and 2002), as expected from Figs. 5 and 6.

The analysis of Figs. 7 and 8 is consistent with a "movie" of TOMS activity which was prepared before the above wave number analysis was developed. It proved to be a useful guide to the study and is available at the following web site: www.usask.ca/physics/isas. The visual identification of motion of the structures is consistent with the quantitative analysis.

It is possible to identify spectral features in Figs. 7 and 8 which are associated with PW that could propagate through to the mesosphere and hence lead to the common activity in the MLT wind-TOMS wavelet products demonstrated in Sect. 4. In three of the four plots for each of 2001 and 2002 there are well-defined maxima (yellow-red mainly), with $\mathrm{m}=1$ (westward) and periods of 10- to 20-d, which are a factor of only 1.4 smaller than the more obvious eastward wave number features. These are likely to be the "normal" mode features, usually considered to dominate the MLT region. It was noted by Lawrence and Randel (1996) that the energy associated with these normal modes is small compared with other stratospheric and tropospheric features. There is also considerable spectral activity in the eastward wave number sectors, with $\mathrm{m}=-1,-2$, and -3 and periods of 10-20 days, which could be associated with the jet stream's variability, fluctuations of the zonal vortex, and PW associated with instability processes. As noted above, it appears possible that such PW may propagate into the eastward circulation of the winter and spring, with comparable ease to the standing PW features, at least to the stratopause. The standing PW are known to be important within the MLT e.g. CIRA 1986. The $m=-2$ spectral features of Manson et al. (2004a) could well be an example of this wave activity.

There is an enormous vertical distance between the $100 \mathrm{mb}$ pressure level near $20 \mathrm{~km}$ that is best associated with the TOMS data and the PW processes inherent within the MFR data $(85 \mathrm{~km})$. Variations in the global temperatures and winds will affect the propagation of the PWs, whose paths are unlikely to be vertical but may involve oblique paths linking $\mathrm{SH}$ and NH. There may indeed be PW sources between the two heights. Thus, correlations between specific features of the wavelets for TOMS and MFR data are not expected to be numerous (as in Sect. 3); and the relationship between the wave number spectra are even more likely to be somewhat tenuous or lacking in exact correspondence. In this latter case the spectra for TOMS involved all longitudes, making it a more global analysis, while the MFR spectra involved only $7000 \mathrm{~km}$ of longitudinal variation. To help bridge the altitude gap, we have just completed a study (Chshyolkova et al., revised, 2005 ${ }^{1}$ ) involving UKMO (United Kingdom Meteorological Office) data-assimilation products (100 and $0.46 \mathrm{mb}$ pressure levels) along with TOMS and MFR data.

\footnotetext{
${ }^{1}$ Chshyolkova, T., Manson, A. H., and Meek, C. E.: Planetary wave coupling processes in the middle atmosphere $(20-90 \mathrm{~km})$ : a study involving TOMS, UKMO and MF radar data, Ann. Geophys., revised, 2005.
}

Summarizing, wave number analysis of the winds (zonal) demonstrates that during winter and at the $50-\mathrm{km}$ level the higher frequency $(<10-d)$ eastward PW modes are largely removed, and eastward and westward PW modes of lower frequency $(>10-d)$ and smaller spatial frequency $(m=+3$ to -3) remain (they then have quite comparable amplitudes). This is consistent with a reviewer's view that such eastward propagating PW may only reach the stratopause. However, the UKMO analysis is also consistent with the general picture of MLT dynamics which emerged from the figures of Sects. 3 and 4.

\section{Further comments and conclusions}

There have been rather extensive discussions throughout the paper, within the sections where the results of the various data analyses have been presented. However, it is useful to make additional comments here on the various wave processes which could be involved in the coupling between the lower stratosphere and mesosphere evident in Figs. 2-6:

a) Propagation of PW from the tropopause-lower stratosphere (circa $20 \mathrm{~km}$ ) into the upper stratosphere (circa $50 \mathrm{~km}$ ) and MLT: as already noted these can be westward propagating "normal" or Rossby waves, which are of small amplitude at the heights near $20 \mathrm{~km}$ most representative of TOMS variability. However other eastward propagating PW of large scale $(\mathrm{m}<4)$, associated with vortex and standing PW variability, along with waves associated with instability processes, have been discussed. On simple physical grounds, based upon the seminal Charney and Drazin (1961) paper, such waves should propagate to the stratopause and their perturbations may reach the MLT. The latter waves require more study based upon GCMs of the most realistic nature; models with tropospheric data-assimilation (e.g. UKMO) are most desirable so that comparisons with MLT observations are more realistic. The sudden stratospheric warmings (SSW) are of great interest since the changing background winds significantly modify PW and tidal propagation. The new SCOSTEP (Scientific Committee on Solar-Terrestrial Physics) program for 2004-8 called CAWSES (Climate and Weather of the Sun-Earth System) will include investigation of these wave processes and the SSW.

b) Nonlinear interactions between PW and tides in either the stratosphere or MLT: instances in our Fig. 6, where regional (North America-Japan sector) products of wavelets for the MFR and TOMS data show spectral maxima at PW periods for the winds and the 12-h tides (for the 16-d PW especially), are indicative of such local interactions in the mesosphere. Wavelet tidal spectra (Manson et al., 2003a, 2004a) are broad at these times, generally consistent with the side-lobes associated with such interactions (e.g. Manson et al., 1982; Mitchell et al., 1996; Pancheva et al., 2002). The latter study was 
very detailed, and provided the zonal wave numbers of the secondary waves, in selected cases. The common features of variability at PW periods in the tidal and TOMS data are evidently complex, however, as the 24-h tide also evidences coupling, but at times which differ from the 12-h tide. The phase differences between the TOMS $(20 \mathrm{~km})$ and MFR $(85 \mathrm{~km})$ data are also highly variable, suggesting a variety of causes and locations for the coupling processes.

Further, we have carefully inspected high resolution spectra for 30-80 day intervals swept throughout the winter months of both years discussed in this paper, and while some spectra reveal side-lobes for the tides at spectral positions, consistent with the simultaneous existence of PW spectral energy, other spectra do not. The issues are clearly complex and warrant detailed and statistical assessments that we will explore elsewhere. Nevertheless, based upon published studies of nonlinear PW-tidal interactions, it appears that this process is a very important one for the modulation of the tides at PW periods.

c) Direct forcing of the tides, following absorption of solar UV, by the temporally and spatially varying stratospheric ozone (associated with PW activity): such processes will provide non migrating tides or components. While migrating tides require several days to provide a stationary global response (Vial et al., 1991), the thermal forcing at PW periods will provide local effects in tidal variability associated with non migrating tides. Standing PW associated with particular regional topographic features, or continent-ocean contrasts, will lead to climatological features in the structure of the total (migrating plus non migrating) tidal fields, as evidenced from satellite data such as HRDI (Manson et al., 2002b, 2004c). Progress in assessing this process will be aided again by modelling, using both GCMs and specific models like GSWM (Global Scale Wave Model). The global and altitudinal distributions of the disturbed ozone and water vapour fields will be necessary to assess the tidal modes forced by these changes; the associated changes in the MLT tidal fields can then be assessed. CAWSES observational and modelling projects in this area are also expected.

d) Gravity waves (GW), modulated by the PW of the stratosphere provide a variable source of GW (e.g. Thayaparan et al., 1995b; Manson et al., 2003b) and their associated effects, such as momentum deposition, within the MLT. Tropospheric and stratospheric waves, especially those associated with active weather processes, will thus provide forcing of the MLT winds and tides at PW periods (Meyer and Forbes, 1997). Such processes will leave a spectral signature on the wavelets used in this study, but the evidence for this process will be difficult to isolate. Progress in this area can also be expected in the near future.
It is expected that all four mechanisms will provide coupling between the PW of the tropopause-lower stratosphere region and the MLT. Of these, the latter three will also produce tidal modulations. The nonlinear PW-tidal interactions are perhaps the easiest to identify, due to the spectral features (temporal and spatial) they create, although the analyses are complex and generally better global coverage of the planet is desirable (space- and ground-based). Some details of these processes and their relative importance will be perhaps best assessed with state-of-the-art GCMs of the lower (with data assimilation) and middle atmosphere, which have both dynamical and chemical processes that are realistically parameterized. Programs such as CAWSES (2004-8) are expected to make considerable contributions to the overall understanding of atmospheric coupling processes.

Acknowledgements. The Canadian authors mention with thanks the support of the NSERC Granting Agency, the University of Saskatchewan through ISAS. The US authors wish to acknowledge the support of the NSF grant ATM-9724570 to the University of Colorado.

Topical Editor U. P. Hoppe thanks C. Jacobi and Y. Portnyagin for their help in evaluating this paper.

\section{References}

Andrews, D. G., Holton, J. R., and Leovy, C. B.: Middle Atmosphere Dynamics, Academic Press, 489, 1987.

Briggs, B. H.: The analysis of spaced sensor records by correlation techniques, in: Handbook for MAP, Vol. 13. SCOSTEP Secr., University of Illinois, Urbana, 166-186, 1984.

Charney, J. G. and Drazin, P. G.: Propagation of planetary-scale disturbances from lower into the upper atmosphere, J. Geophys. Res., 66, 83-109, 1961.

Fedulina, I. N., Pogoreltsev, A. ., and Vaughan, G.: Seasonal, interannual and short-term variability of planetary waves in UKMO assimilated fields, Q.J.R. Meteorol. Soc., 128, 1-999, 2002.

Fusco, A. C. and Salby, M. L.: Interannual Variations of Total Ozone and Their Relationship to Variations of Planetary Wave Activity, American Meteorol. Soc., 12, 1619-1629, 1999.

Gregory, J. B. and Manson, A. H.: Winds and Wave Motions to $110 \mathrm{~km}$ at Mid-Latitudes, III. Response of Mesospheric and Thermospheric Winds to Major Stratospheric Warmings, J. of Atmospheric Sciences, 32, 1676-1681, 1975.

Hagan, M. E.: Comparative effects of migrating solar sources on tidal signatures in the middle and upper atmosphere, J. Geophys. Res., 101, 21 213-21 222, 1996.

Hoffmann, P., Singer, W., and Keuer, D.: Variability of the mesospheric wind field at middle and Arctic latitudes in winter and its relation to stratospheric circulation disturbances, J. Atmos. Solar-Terr. Phys., 64, 1229-1240, 2002.

Igarashi, K., Nishimuta, Y, Murayama, Y., Tsuda, T., Nakamura T., and Tsutsumi, M.: Comparison of wind measurements between the Yamagawa MF radar and the MU radar, Geophys. Res. Lett., 23, 3341-3344, 1996.

Jacobi, Ch., Lange, R., Kurschner, D., Manson, A. H., and Meek, C. E.: A long-term comparison of Saskatoon MF radar and Collm LF D1 mesosphere-lower thermosphere wind measurements, Physics and Chemistry of the Earth, Part C, 26, 419-424, 2001. 
Jacobi, Ch., Kurschner, D., Muller, H. G., Pancheva, D., Mitchell, N. J., and Naujokat, B.: response of the mesopause region dynamics to the February 2001 stratospheric warming, J. Atmos. Solar-Terr. Phys., 65, 843-855, 2003.

Kumar, P. and Foufoula-Georgiou, E.: Wavelet analysis for geophysical applications, Rev. Geophysics, 35, 385-412, 1997.

Lawrence, A. R. and Jarvis, M. J.: Simultaneous observations of planetary waves from 30 to $220 \mathrm{~km}$, J. Atmos. Solar-Terr. Phys., 65, 765-777, 2003.

Lawrence, B. N. and Randel, W. J.: Variability in the mesosphere observed by the Nimbus 6 pressure modulator radiometer, J. Geophys. Res., 101, 23 475-23 489, 1996.

Lomb, N. R.: Least-squares frequency analysis of unequally spaced data Astrophys. Space Sci, 39, 447-462, 1976.

Luo, Y., Manson, A. H., Meek, C. E., Meyer, C. K., and Forbes, J. M.: The quasi 16-day oscillations in the mesosphere and lower thermosphere at Saskatoon (52 N, 107 W), 1980-1996, J. Geophys. Res., 105, 2125-2138, 2000.

Luo, Y., Manson, A. H., Meek, C. E., Igarashi, K., and Jacobi, Ch.: Extra long period (20-40 day) oscillations in the mesospheric and lower thermospheric winds: observations in Canada, Europe and Japan, and considerations of possible solar influences, J. Atmos. Solar-Terr. Phys., 63, 835-852, 2001.

Luo, Y., Manson, A. H., Meek, C. E., Meyer, C. K., Forbes, J. M., Burrage, M. D., Fritts, D. C., Hall, C. M., Hocking, W. K., MacDougall, J., Riggin, D. M., and Vincent, R. A.: The 16-day planetary waves: multi-MF radar observations from the arctic to equator and comparisons with the HRDI measurements and the GSWM modeling results, Ann. Geophys., 20, 691-709, 2002a, SRef-ID: 1432-0576/ag/2002-20-691.

Luo, Y., Manson, A. H., Meek, C. E., Thayaparan, T., MacDougall, J., and Hocking, W.: The 16-day wave in the mesosphere and lower thermosphere: simultaneous observations at Saskatoon (52 N, $107 \mathrm{~W})$ and London (43 N, $81 \mathrm{~W})$, Canada, J. Atmos. Solar-Terr. Phys., 64, 1287-1307, 2002 b.

Manson, A. H., Meek, C. E., Gregory, J. B., and Chakrabarty, D. K.: Fluctuations in Tidal (24-, 12-h) Characteristics and Oscillations (8-h-5-d) in the Mesosphere and Lower Thermosphere (70$110 \mathrm{~km}$ ): Saskatoon (52 N, $107 \mathrm{~W}), 1979-1981$; Planet Space Sci. 30, 12, 1283-1294, 1982.

Manson, A. H., Meek, C. E., Brekke, A., and Moen, J.: Mesosphere and lower thermosphere $(80-120 \mathrm{~km})$ winds and tides from near Tromso (70 N, $19 \mathrm{E}$ ): comparisons between radars (MF, EISCAT, VHF) and rockets, J. Atmos. Terr. Phys. 54, 927-950, 1992.

Manson, A. H., Yi, F., Hall, C., and Meek, C. E.: Comparisons between instantaneous wind measurements made at Saskatoon $(52 \mathrm{~N}, 107 \mathrm{~W})$ using the co-located medium frequency radars and Fabry-Perot interferometer instruments: climatologies (19881992) and case studies, J. Geophys. Res. 101, 29553-29563, 1996.

Manson, A. H., Meek, C. E., Stegman, J., Espy, P. J., Roble, R. G., Hall, C. M.: Hoffmann, P., and Jacobi, Ch.: Springtime transitions in mesopause airglow and dynamics: photometer and MF radar observations in the Scandinavian and Canadian sectors, J. Atmos. Terr. Phys. 64, 1131-1146, 2002a.

Manson, A. H, Luo, Y., and Meek, C. E.: Global Distributions of Diurnal and Semi- Diurnal Tides: Observations from HRDIUARS of the MLT Region, Ann. Geophys., 20, 1877-1890, 2002b,

SRef-ID: 1432-0576/ag/2002-20-1877.

Manson, A. H., Meek, C. E., Avery, S. K., and Thorsen, D.: Ionospheric and dynamical characteristics of the mesosphere-lower thermosphere region over Platteville $\left(40^{\circ} \mathrm{N}, 105^{\circ} \mathrm{W}\right)$ and comparisons with the region over Saskatoon $\left(52^{\circ} \mathrm{N}, 107^{\circ} \mathrm{W}\right)$. J. Geophys. Res., 108, D13, 4398, doi:10.1029/2002JD002835, 2003 a. Manson, A. H., Meek, C. E., Luo, Y., Hocking, W. K., MacDougall, J., Riggin, D., Fritts, D. C., and Vincent, R. A.: Modulation of gravity waves by planetary waves ( 2 and $16 \mathrm{~d}$ ): observations with the North American-Pacific MLT-MFR radar network. J. Atmos. Solar-Terr. Phys., 65, 85-104, 2003b.

Manson, A. H., Meek, C. E., Chshyolkova, T., Avery, S. K., Thorsen, D., MacDougall, J. W., Hocking, W., Murayama, Y., Igarashi, K., Namboothiri, S. P., and Kishore, P.: Longitudinal and Latitudinal Variations in Dynamic Characteristics of the MLT (70-95 km): A Study Involving the CUJO network; Ann. Geophys., 22, 347-365, 2004a.

Manson, A. H., Meek, C. E., Hall, C. M., Nozawa, S., Mitchell, N. J., Pancheva, D., Singer, W., and Hoffmann, P.: Mesopause Dynamics from the Scandinavian Triangle of Radars within the PSMOS-DATAR Project; Ann. Geophys., 22, 367-386, 2004b.

Manson, A. H., Meek, C. E., Hagan, M., Zhang, X., and Luo, Y.: Global Distributions of Diurnal and Semi-Diurnal Tides: Observations from HRDI-UARS of the MLT Region and Comparisons with GSWM-02 (migrating, non-migration modes), Ann. Geophys., 22, 1529-1548, 2004c,

SRef-ID: 1432-0576/ag/2004-22-1529.

Meek, C. E.: An efficient method for analyzing ionospheric drifts data, J. Atmos. Terr. Phys. 42, 835-839, 1980.

Meek, C. E., Manson, A. H., Burrage, M. D., Garbe, G., and Cogger, L. L.: Comparisons between Canadian prairie MF radars, FPI (green and OH lines) and UARS HRDI systems, Ann. Geophys., 15, 1099-1110, 1997,

SRef-ID: 1432-0576/ag/1997-15-1099.

Meyer, C. K., and Forbes, J. M.: Natural oscillations of the ionosphere-thermosphere-mesosphere (ITM) system, J. Atmos. Solar-Terr. Phys., 59, 2185-2202, 1997a.

Mitchell, N., Williams, P. J. S., Beard, A. G., Buesnel, G., and Muller, H.: Non-linear planetary/ tidal wave interactions in the lower thermosphere observed by meteor radar, Ann. Geophys., 19, 364-366, 1996.

Murayama, Y., Igarashi, K., Rice, D., Watkins, B., Collins, R., Mizutani, K., Saito, Y., and Kainuma, S.: Medium Frequency Radars in Japan and Alaska, for Upper Atmosphere Observations, IEICE Trans., 1996-2003, E83-B, 2000.

Pancheva, D., Merzlyakov, E., Mitchell, N. J., Portnyagin, Yu., Manson, A. H., et al.: Global-scale tidal variability during the PSMOS campaign of June-August 1999: interaction with planetary waves, J. Atmos. Solar-Terr. Phys. 64, 1865-1896, 2002.

Pancheva, D., Mitchell, N., Middleton, H. and Muller, H.: Variability of the semidiurnal tide due to fluctuations in solar activity and total ozone, J. Atmos. Solar-Terr. Phys. 65, 1-19, 2003.

Pogoreltsev, A. I., Fedulina, I. N., Mitchell, N. J., Muller, H. G., Luo, Y., Meek, C. E. and Manson, A. H.: Global free oscillations of the atmosphere and secondary planetary waves in the mesosphere and lower thermosphere region during August/September time conditions, J. Geophys. Res., 107 (D24), 4799, doi:10.1029/2001JD001535, 2002.

Portnyagin, Y. I. and Solovjova, T. V.: Global empirical wind model for the upper mesosphere/lower thermosphere, I. Prevailing wind, Ann. Geophys., 18, 300-315, 2000,

\section{SRef-ID: 1432-0576/ag/2000-18-300}

Scargle, J. D.: Studies in astronomical time series analysis, II. Statistical aspects of spectral analysis of unevenly spaced data, Astrophsy. J., 263, 835-853, 1982. 
Schoeberl, M. R. and Krueger A. J.: Medium Scale Disturbances In Total Ozone During Southern Hemisphere Summer: Bulletin of the American Meterological Society, 64, No. 12, December 1983.

Thayaparan, T., Hocking, W. K., and MacDougall, J.: Middle atmospheric winds and tides over London, Canada $\left(43^{\circ} \mathrm{N}, 81^{\circ} \mathrm{W}\right)$ during 1992-1993, Radio Sci., 30, 1293-1309, 1995a.

Thayaparan, T., Hocking, W. K., and MacDougall, J.: Observational evidence of tidal/gravity wave interactions using the UWO $2 \mathrm{MHz}$ radar. Geophys. Res. Letters 22, 373-376, $1995 \mathrm{~b}$.

Thayaparan, T., Hocking, W. K., MacDougall, J., Manson, A. H., and Meek, C. E.: Simultaneous observations of the 2-day wave at London $\left(43^{\circ} \mathrm{N}, 81^{\circ} \mathrm{W}\right)$ and Saskatoon $\left(52^{\circ} \mathrm{N}, 107^{\circ} \mathrm{W}\right)$ near $91 \mathrm{~km}$ altitude during the two years of 1993 and 1994, Ann. Geophys., 15, 1324-1339, 1997,

SRef-ID: 1432-0576/ag/1997-15-1324
Thayaparan, T. and Hocking, W. K.: A long-term comparison of winds and tides measured at London, Canada $\left(43^{\circ} \mathrm{N}, 81^{\circ} \mathrm{W}\right)$ by co-located MF and meteor radars during 1994-1999, J. Atmos. Terr. Phys. 64, 931-946, 2002.

Venne, D. E. and Stanford, J. L.: An observational study of highlatitude stratospheric planetary-waves in winter, J. Atmos Sci. 39, 1026-1034, 1982.

Vial, F., Forbes, J. M., and Miyahara, S.: Some Transient Aspects of Tidal Propagation, J. Geophys. Res., 96 (A2), 1215-1224, 1991. 